\title{
The Cauchy-Dirichlet Problem for a Class of Linear Parabolic Differential Equations with Unbounded Coefficients in an Unbounded Domain
}

\author{
Gerardo Rubio \\ Comisión Nacional de Seguros y Fianzas, Distrito Federal, Mexico \\ Correspondence should be addressed to Gerardo Rubio, grubioh@yahoo.com \\ Received 20 December 2010; Revised 18 March 2011; Accepted 19 April 2011 \\ Academic Editor: Lukasz Stettner
}

Copyright (C) 2011 Gerardo Rubio. This is an open access article distributed under the Creative Commons Attribution License, which permits unrestricted use, distribution, and reproduction in any medium, provided the original work is properly cited.

We consider the Cauchy-Dirichlet problem in $[0, \infty) \times D$ for a class of linear parabolic partial differential equations. We assume that $D \subset \mathbb{R}^{d}$ is an unbounded, open, connected set with regular boundary. Our hypotheses are unbounded and locally Lipschitz coefficients, not necessarily differentiable, with continuous data and local uniform ellipticity. We construct a classical solution to the nonhomogeneous Cauchy-Dirichlet problem using stochastic differential equations and parabolic differential equations in bounded domains.

\section{Introduction}

In this paper, we study the existence and uniqueness of a classical solution to the CauchyDirichlet problem for a linear parabolic differential equation in a general unbounded domain. Let $\mathcal{L}$ be the differential operator

$$
\mathcal{L}[u](t, x):=\sum_{i, j=1}^{d} a_{i j}(t, x) D_{i j} u(t, x)+\sum_{i=1}^{d} b_{i}(t, x) D_{i} u(t, x),
$$

where $\left\{a_{i j}\right\}=a=\sigma \sigma^{\prime}, D_{i}=\partial / \partial x_{i}$, and $D_{i j}=\partial^{2} / \partial x_{i} \partial x_{j}$. The Cauchy-Dirichlet problem is

$$
-u_{t}(t, x)+\mathcal{L}[u](t, x)+c(t, x) u(t, x)=-f(t, x), \quad(t, x) \in(0, \infty) \times D,
$$




$$
\begin{gathered}
u(0, x)=h(x), \quad x \in D, \\
u(t, x)=g(t, x), \quad(t, x) \in(0, \infty) \times \partial D,
\end{gathered}
$$

where $D \subset \mathbb{R}^{d}$ is an unbounded, open, connected set with regular boundary.

In the case of bounded domains, the Cauchy-Dirichlet problem is well understood (see $[1,2]$ for a detailed description of this problem). Moreover, when the domain is unbounded and the coefficients are bounded, the existence of a classical solution to (1.2) is well known. For a survey of this theory see $[3,4]$ where the problem is studied with analytical methods and [5] for a probabilistic approach.

In the last years, parabolic equations with unbounded coefficients in unbounded domains have been studied in great detail. For the particular case when $D=\mathbb{R}^{d}$, there exist many papers in which the existence, uniqueness, and regularity of the solution is studied under different hypotheses on the coefficients; see for example, [6-17].

In the case of general unbounded domains, Fornaro et al. in [18] studied the homogeneous, autonomous Cauchy-Dirichlet problem. They proved, using analytical methods in semigroups, the existence and uniqueness of a solution to the Cauchy-Dirichlet problem when the coefficients are locally $C^{1, \alpha}$, with $a_{i j}$ bounded, $b$ and $c$ functions with a Lyapunov type growth; that is, there exists a function $\varphi \in C^{1,2}\left((0, T) \times \mathbb{R}^{d}\right)$ such that

$$
\lim _{|x| \rightarrow \infty} \inf _{0 \leq t \leq T} \varphi(t, x)=\infty
$$

and for some $\lambda>0$,

$$
\sup _{[0, T] \times \mathbb{R}^{d}}\left\{\left(-\frac{\partial}{\partial t}+\mathcal{L}\right) \varphi(t, x)-\lambda \varphi(t, x)\right\}<\infty
$$

It is also assumed that $D$ has a $C^{2}$ boundary. Schauder-type estimates were obtained for the gradient of the solution in terms of the data. Bertoldi and Fornaro in [19] obtained analogous results for the Cauchy-Neumann problem for an unbounded convex domain. Later, in [20] Bertoldi et al. generalized the method to nonconvex sets with $C^{2}$ boundary. They studied the existence, uniqueness, and gradient estimates for the Cauchy-Neumann problem. For a survey of this results, see [21].

Using the theory of semigroups, Da Prato and Lunardi studied, in [22, 23], the realization of the elliptic operator $A=(1 / 2) \Delta-\langle D U, D \cdot\rangle$, in the functions spaces $L^{2}(D)$, $L^{\infty}(D)$ and $C_{b}(D)$, when $U$ is an unbounded convex function defined in a convex set $D$. They proved existence and uniqueness for the elliptic and parabolic equations associated with $\mathcal{A}$ and studied the regularity of the semigroup generated by $\mathcal{A}$. Geissert et al. in [24], made a similar approach for the Ornstein-Uhlenbeck operator.

In the paper of Hieber et al. [25], the existence and uniqueness of a classical solution for the autonomous, nonhomogeneous Cauchy-Dirichlet and Cauchy-Neumann problems is proved. The domain is considered to be an exterior domain with $C^{3}$ boundary. The coefficients are assumed to be $C^{3, \alpha}$ continuous functions with Lyapunov type growth. The continuity properties of the semigroup generated by the solution of the parabolic problem are studied in the spaces $C_{b}(\bar{D})$ and $L^{p}(D)$. 
In all the papers cited above, the uniformly elliptic condition is assumed; that is, there exists $\lambda>0$ such that for all $(t, x) \in[0, \infty) \times \bar{D}$ we have that $\sum a_{i j}(t, x) \eta_{i} \eta_{j} \geq \lambda\|\eta\|^{2}$ for all $\eta \in \mathbb{R}^{d}$.

In this paper, we prove the existence and uniqueness of a classical solution to (1.2), when the coefficients are locally Lipschitz continuous in $x$ and locally Hölder continuous in $t, a_{i j}$ has a quadratic growth, $b_{i}$ has linear growth, and $c$ is bounded from above. We allow $f, g$, and $h$ to have a polynomial growth of any order. We also consider the elliptic condition to be local; that is, for any $[0, T] \times A \subset[0, \infty) \times \bar{D}$, there exists $\lambda(T, A)$ such that $\sum a_{i j}(t, x) \eta_{i} \eta_{j} \geq \lambda(T, A)\|\eta\|^{2}$ for all $t \in[0, T], x \in A$ and $\eta \in \mathbb{R}^{d}$. We assume that $D$ is an unbounded, connected, open set with regular boundary (see [1] Chapter III, Section 4, for a definition of regular boundary). Furthermore, we prove that the solution is locally Hölder continuous up to the second space derivative and the first time derivative.

Our approach is using stochastic differential equations and parabolic differential equations in bounded domains. For proving existence, many analytical methods construct the solution by solving the problem in nested bounded domains that approximate the domain $D$. In these cases, the convergence of the approximating solutions is always a very difficult task. Unlike these methods, first we propose, as a solution to (1.2), a functional of the solution to a SDE,

$$
\begin{aligned}
v(t, x)= & \mathbb{E}_{x}\left[\int_{0}^{t \wedge \tau_{D}} e^{\int_{0}^{s} c(t-r, X(r)) d r} f(t-s, X(s)) d s\right] \\
& +\mathbb{E}_{x}\left[e^{\int_{0}^{t} c(t-r, X(r)) d r} h(X(t)) \mathbb{1}_{\tau_{D} \geq t}\right] \\
& +\mathbb{E}_{x}\left[e^{\int_{0}^{\tau_{D}} c(t-r, X(r)) d r} g\left(t-\tau_{D}, X\left(\tau_{D}\right)\right) \mathbb{1}_{\tau_{D}<t}\right]
\end{aligned}
$$

where

$$
\begin{gathered}
d X(s)=b(t-s, X(s)) d s+\sigma(t-s, X(s)) d W(s), \quad X(0)=x, \\
\tau_{D}:=\inf \{s>0 \mid X(s) \notin \bar{D}\} .
\end{gathered}
$$

Using the continuity of the paths of the SDE, we prove that this function is continuous in $[0, \infty) \times \bar{D}$. Then, using the theory of parabolic equations in bounded domains, we study locally the regularity of the function $v$ and prove that it is a $C^{1,2}$ function. Finally, with some standard arguments, we prove that it solves the Cauchy-Dirichlet problem. This kind of idea has been used for several partial differential problems (see [5, 26, 27]).

In Section 2, we introduce the notation and the hypotheses used throughout the paper. Section 3 presents the main result. In this section we prove that if the function $v$ is smooth, then it has to be the solution to the Cauchy-Dirichlet problem. Section 4 is devoted to prove the required differentiability for the candidate function. Finally, in Section 5, the reader will find some of the results used in the proof of our main theorem. 


\section{Preliminaries and Notation}

In this section, we present the hypotheses and the notation used in this paper.

\subsection{Domain}

Let $D \subset \mathbb{R}^{d}$ be an unbounded, open, connected set with boundary $\partial D$ and closure $\bar{D}$. We assume that $D$ has a regular boundary; that is, for any $x \in \partial D, x$ is a regular point (see [1] Chapter III, Section 4 or [28] Chapter 2, Section 4, for a detailed discussion of regular points). We denote the hypotheses on $D$ by $\mathbf{H O}$.

\subsection{Stochastic Differential Equation}

Let $\left(\Omega, \mathcal{F}, \mathbb{P},\left\{\mathcal{F}_{s}\right\}_{s \geq 0}\right)$ be a complete filtered probability space and let $\{W\}=\left\{W_{i}\right\}_{i=1}^{d}$ be a $d$ dimensional brownian motion defined in it. For $t \geq 0$ and $x \in \bar{D}$, consider the stochastic differential equation

$$
d X(s)=b(t-s, X(s)) d s+\sigma(t-s, X(s)) d W(s), \quad X(0)=x,
$$

where $b=\left\{b_{i}\right\}_{i=1}^{d}$ and $\sigma=\left\{\sigma_{i j}\right\}_{i, j=1}^{d}$. Although this process is the natural one for solving equation (1.2), it does not posses many good properties. The continuity of the flow process does not imply the continuity with respect to $t$. Furthermore, although this process is a strong Markov process, it is not homogeneous in time, a very useful property for proving the results in this paper.

To overcome these difficulties, we augment the dimension considering the following process

$$
d \xi(s)=-d s, \quad \xi(0)=t
$$

Then the process $\{\xi(s), X(s)\}$ is solution to

$$
\begin{gathered}
d \xi(s)=-d s, \\
d X(s)=b(\xi(s), X(s)) d s+\sigma(\xi(s), X(s)) d W(s),
\end{gathered}
$$

with $(\xi(0), X(0))=(t, x)$. Throughout this paper we will use both processes, $X(s)$ and $(\xi(s), X(s))$, in order to simplify the exposition. For the expectation, we use the notation

$$
\mathbb{E}_{x}[\cdot]:=\mathbb{E}[\cdot \mid X(0)=x]
$$

when considering the process $X$ defined as in (2.1) and the notation

$$
\mathbb{E}_{t, x}[\cdot]:=\mathbb{E}[\cdot \mid(\xi(0), X(0))=(t, x)]
$$

when working with the joint process $(\xi, X)$ defined in (2.3). 
We need to define the following stopping times

$$
\begin{gathered}
\tau_{D}:=\inf \{s>0 \mid X(s) \notin \bar{D}\}, \\
\tau:=\tau_{D} \wedge t .
\end{gathered}
$$

Remark 2.1. Observe that $\tau$ is the exit time of the process $(\xi(s), X(s))$ from the set $[0, \infty) \times \bar{D}$, that is,

$$
\tau=\inf \{s>0 \mid(\xi(s), X(s)) \notin[0, \infty) \times \bar{D}\}
$$

We cannot guarantee that the process $X(s)$ leaves the set $D$ in a finite time however, the process $\xi(s)$ reaches the boundary $s=0$ at time $t$. Thus, the joint process $(\xi(s), X(s))$ leaves the set $[0, \infty) \times \bar{D}$ in a bounded time.

We assume the following hypotheses on the coefficients $b$ and $\sigma$. We denote them by H1. The matrix norm considered is $\|\sigma\|^{2}:=\operatorname{tr} \sigma \sigma^{\prime}=\sum_{i, j} \sigma_{i j}^{2}$.

H1.

Let

$$
\begin{gathered}
\sigma: \mathbb{R} \times \mathbb{R}^{d} \longrightarrow \mathcal{M}\left(\mathbb{R}^{d} \times \mathbb{R}^{d}\right) \\
b: \mathbb{R} \times \mathbb{R}^{d} \longrightarrow \mathbb{R}^{d}
\end{gathered}
$$

be continuous functions such that

(1) Continuity. Let $\lambda \in(0,1)$. For all $T>0, n \geq 1$, there exists $L_{1}(T, n)$ such that

$$
\|\sigma(r, x)-\sigma(s, y)\|^{2}+\|b(r, x)-b(s, y)\|^{2} \leq L_{1}(T, n)^{2}\left(|r-s|^{2 \lambda}+\|x-y\|^{2}\right)
$$

for all $|r|,|s| \leq T,\|x\| \leq n,\|y\| \leq n$.

(2) Linear-Growth. For each $T>0$, there exists a constant $K_{1}(T)$ such that

$$
\|\sigma(r, x)\|^{2}+\|b(r, x)\|^{2} \leq K_{1}(T)^{2}\left(1+\|x\|^{2}\right)
$$

for all $|r| \leq T, x \in \mathbb{R}^{d}$.

(3) Local Ellipticity. Let $A \subset \bar{D}$ be any bounded, open, connected set and $T>0$. There exists $\lambda(T, A)>0$ such that, for all $(r, x) \in[0, T] \times \bar{A}$ and $\eta \in \mathbb{R}^{d}$,

$$
\sum_{i, j} a_{i j}(r, x) \eta_{i} \eta_{j} \geq \lambda(T, A)\|\eta\|^{2}
$$

where $\left\{a_{i j}\right\}=a=\sigma \sigma^{\prime}$. 
Remark 2.2. Observe that the local ellipticity is only assumed on $[0, \infty) \times \bar{D}$. This condition is used to prove the existence of a classical solution to (1.2) and so is only needed in that set. The local Lipschitz condition and the linear growth are assumed on $\mathbb{R} \times \mathbb{R}^{d}$ to ensure the existence of a strong solution to (2.3) for $s \in[0, \infty)$.

Remark 2.3. If we assume, in the more natural case, that

$$
\begin{gathered}
\sigma:[0, \infty) \times \mathbb{R}^{d} \longrightarrow \mathcal{M}\left(\mathbb{R}^{d} \times \mathbb{R}^{d}\right), \\
b:[0, \infty) \times \mathbb{R}^{d} \longrightarrow \mathbb{R}^{d}
\end{gathered}
$$

are continuous functions satisfying the hypotheses in $\mathbf{H 1}$ restricted to the set $[0, \infty) \times \mathbb{R}^{d}$, then we can extend them to be defined for negative values of $r$ as follows: let $\widehat{b}$ and $\widehat{\sigma}$ be defined as

$$
\begin{aligned}
& \widehat{b}(r, x)= \begin{cases}b(r, x) & \text { if } r \geq 0, \\
b(0, x) & \text { if } r<0\end{cases} \\
& \widehat{\sigma}(r, x)= \begin{cases}\sigma(r, x) & \text { if } r \geq 0 \\
\sigma(0, x) & \text { if } r<0\end{cases}
\end{aligned}
$$

It is easy to see that these functions satisfy $\mathbf{H} 1$ with the same constants $L_{1}$ and $K_{1}$.

Remark 2.4. It follows, from the nondegeneracy (the local ellipticity) of the process $X(s)$, the regular boundary of the set $D$ and Lemma 4.2, Chapter 2 in [28], that, for any $t, x \in[0, \infty) \times \bar{D}$,

$$
\mathbb{P}_{t, x}\left[\tau=\tau^{\prime}\right]=1
$$

where $\tau^{\prime}:=\inf \{s>0 \mid(\xi(s), X(s)) \notin(0, \infty) \times D\}$ (see Remark 2.1). this work.

The next proposition presents some of the properties of the process $(\xi, X)$ required in

Proposition 2.5. As a consequence of $\mathbf{H 1},(\xi, X)$ has the following properties.

(i) For all $(t, x) \in[0, \infty) \times \mathbb{R}^{d}$, there exists a unique strong solution to (2.3).

(ii) The process $\{\xi(s), X(s)\}_{s \geq 0}$ is a strong homogeneous Markov process.

(iii) The process $\{\xi(s), X(s)\}_{s \geq 0}$ does not explode in finite time a.s..

(iv) For all $x \in \mathbb{R}^{d}, T>0$, and $r \geq 1$,

$$
\mathbb{E}_{x}\left[\sup _{0 \leq s \leq T}\|X(s)\|^{2 r}\right] \leq C\left(T, K_{1}, r\right)\left(1+\|x\|^{2 r}\right) .
$$


Proof. See

(i) Theorem 1.1 Chapter V in [26] or Theorem 3.2, Chapter 6 in [29].

(ii) Theorem 4.6, Chapter 5 in [27] or Proposition 3.15, Chapter 6 in [29].

(iii) Theorem 10.2.2 in [30].

(iv) Theorem 2.3, Chapter 5 in [31] or Corollary 3.3, Chapter 6 in [29].

\subsection{The Cauchy-Dirichlet Problem}

Consider the following differential operator:

$$
\mathcal{L}[u](t, x):=\sum_{i, j=1}^{d} a_{i j}(t, x) D_{i j} u(t, x)+\sum_{i=1}^{d} b_{i}(t, x) D_{i} u(t, x),
$$

where $D_{i}=\partial / \partial x_{i}, D_{i j}=\partial^{2} / \partial x_{i} \partial x_{j}$, and $\left\{a_{i j}\right\}_{i, j=1}^{d}=a=\sigma \sigma^{\prime}$. For the rest of the paper, we assume that the coefficients of $\mathcal{L}$ satisfy $\mathbf{H 1}$.

The Cauchy-Dirichlet problem for a linear parabolic equation is

$$
\begin{gathered}
-u_{t}(t, x)+\mathcal{L}[u](t, x)+c(t, x) u(t, x)=-f(t, x), \quad(t, x) \in(0, \infty) \times D, \\
u(0, x)=h(x), \quad x \in D, \\
u(t, x)=g(t, x), \quad(t, x) \in(0, \infty) \times \partial D .
\end{gathered}
$$

We assume the following hypotheses for the functions $c, f, h$, and $g$. We denote them by $\mathbf{H} \mathbf{2}$.

H2.

(1) Let

$$
\begin{aligned}
& c:[0, \infty) \times \bar{D} \longrightarrow \mathbb{R}, \\
& f:[0, \infty) \times \bar{D} \longrightarrow \mathbb{R}
\end{aligned}
$$

be continuous functions such that

(i) Continuity. Let $\lambda \in(0,1)$. For all $T>0, n \geq 1$, there exists a constant $L_{2}(T, n)$ such that

$$
\begin{aligned}
& \|f(r, x)-f(s, y)\|^{2}+\|c(r, x)-c(s, y)\|^{2} \leq L_{2}(T, n)^{2}\left(|r-s|^{2 \lambda}+\|x-y\|^{2}\right), \\
& \text { for all } 0 \leq s, r \leq T, x, y \in \bar{D} \text { with }\|x\| \leq n,\|y\| \leq n . \\
& \text { (ii) Growth. There exists } c_{0} \geq 0 \text { such that }
\end{aligned}
$$

$$
c(r, x) \leq c_{0} \quad \forall(r, x) \in[0, \infty) \times \bar{D} .
$$


There exists $k>0$, such that for all $T>0$, a constant $K_{2}(T)$ exists such that

$$
|f(r, x)| \leq K_{2}(T)\left(1+\|x\|^{k}\right)
$$

for all $0 \leq r \leq T, x \in \bar{D}$

(2) Let

$$
\begin{gathered}
h: \bar{D} \longrightarrow \mathbb{R}, \\
g:[0, \infty) \times \partial D \longrightarrow \mathbb{R}
\end{gathered}
$$

be continuous functions such that

(i) Growth. There exists $k>0$, such that, for all $T>0$, there exists a constant $K_{3}(T)$ such that

$$
|h(x)|+|g(r, x)| \leq K_{3}(T)\left(1+\|x\|^{k}\right)
$$

for all $(r, x) \in[0, T] \times \bar{D}$.

(ii) Consistency. There exists consistency in the intersection of the space and the time boundaries, that is,

$$
h(x)=g(0, x)
$$

for $x \in \partial D$.

\subsection{Additional Notation}

If $\mu$ is a locally Lipschitz function defined in some set $R$, then, for any bounded open set $A$ for which $\bar{A} \subset R$, we denote, by $K_{\mu}(A)$ and $L_{\mu}(A)$, the constants

$$
\begin{gathered}
K_{\mu}(A):=\sup _{x \in A}\|\mu(x)\|<\infty, \\
L_{\mu}(A):=\sup _{x, y \in A, x \neq y} \frac{\|\mu(x)-\mu(y)\|}{\|x-y\|}<\infty .
\end{gathered}
$$

If $v:[0, \infty) \rightarrow \mathbb{R}^{d}$, then, for all $T>0$,

$$
\|v\|_{T}:=\sup _{0 \leq s \leq T}\|v(s)\|
$$

The space $C_{\text {loc }}^{1,2, \lambda}((0, \infty) \times D)$ is the space of all functions such that they and all their derivatives up to the second order in $x$ and first order in $t$, are locally Hölder continuous of order $\lambda$. 


\section{Main Result}

In this section, we present the main result of this work and some parts of the proof.

Theorem 3.1. Assume $\mathbf{H 0}, \mathbf{H 1}$, and $\mathbf{H 2}$. Then, there exists a unique solution $u \in C([0, \infty) \times \bar{D}) \cap$ $C_{\text {loc }}^{1,2, \lambda}((0, \infty) \times D)$ to $(2.17)$. The solution has the representation

$$
\begin{aligned}
u(t, x)= & \mathbb{E}_{x}\left[\int_{0}^{t \wedge \tau_{D}} e^{\int_{0}^{s} c(t-r, X(r)) d r} f(t-s, X(s)) d s\right] \\
& +\mathbb{E}_{x}\left[e^{\int_{0}^{t} c(t-r, X(r)) d r} h(X(t)) \mathbb{1}_{\tau_{D} \geq t}\right] \\
& +\mathbb{E}_{x}\left[e^{\int_{0}^{\tau_{D}} c(t-r, X(r)) d r} g\left(t-\tau_{D}, X\left(\tau_{D}\right)\right) \mathbb{1}_{\tau_{D}<t}\right],
\end{aligned}
$$

where $X$ is the solution to the stochastic differential equation

$$
\begin{gathered}
d X(s)=b(t-s, X(s)) d s+\sigma(t-s, X(s)) d W(s), \quad X(0)=x, \\
\tau_{D}:=\inf \{s>0 \mid X(s) \notin \bar{D}\} .
\end{gathered}
$$

Furthermore, for all $T>0$,

$$
\sup _{0 \leq t \leq T}|u(t, x)| \leq C\left(T, c_{0}, K_{1}, K_{2}, K_{3}, k\right)\left(1+\|x\|^{k}\right), \quad x \in \bar{D}
$$

where $c_{0}, K_{1}, K_{2}, K_{3}$, and $k$ are the constants defined in $\mathbf{H 1}$ and $\mathbf{H} \mathbf{2}$.

The proof of this theorem is given by several lemmas. The method we will use has the following steps: first we define a functional of the process $X$ as a candidate solution. Let $v:[0, \infty) \times \bar{D} \rightarrow \mathbb{R}$ be defined as

$$
\begin{aligned}
v(t, x):= & \mathbb{E}_{x}\left[\int_{0}^{t \wedge \tau_{D}} e^{\int_{0}^{s} c(t-r, X(r)) d r} f(t-s, X(s)) d s\right] \\
& +\mathbb{E}_{x}\left[e^{\int_{0}^{t} c(t-r, X(r)) d r} h(X(t)) \mathbb{1}_{\tau_{D} \geq t}\right] \\
& +\mathbb{E}_{x}\left[e^{\int_{0}^{\tau_{D}} c(t-r, X(r)) d r} g\left(t-\tau_{D}, X\left(\tau_{D}\right)\right) \mathbb{1}_{\tau_{D}<t}\right] .
\end{aligned}
$$

If $v \in C([0, \infty) \times \bar{D}) \cap C^{1,2}((0, \infty) \times D)$, then there exist some standard arguments (see [27, chapter 4]) to prove that $v$ is the unique solution to (2.17). The rest of this section is devoted to proving Theorem 3.1 in the case when $v$ is a "regular" function. The proof is divided into two lemmas: the first one proves that if $v \in C([0, \infty) \times \bar{D}) \cap C^{1,2}((0, \infty) \times D)$, then $v$ is a solution to equation. The second one proves that in case of existence of a classical solution, $u$, to problem (2.17), then it is unique and has the form given by $v$ in (3.4). The regularity of $v$ is proved in Section 4 below. 
The next proposition gives an extension of the boundary data to all the space $[0, \infty) \times$ $\mathbb{R}^{d}$. This extension is given to simplify the notation and is required in the proofs of Lemmas 4.2 and 4.3 .

Proposition 3.2. Assume H2. Then, there exists a continuous function $G:[0, \infty) \times \mathbb{R}^{d} \rightarrow \mathbb{R}$ such that

$$
\begin{gathered}
G(t, x)=g(t, x), \quad(t, x) \in[0, \infty) \times \partial D, \\
G(0, x)=h(x), \quad x \in \bar{D} .
\end{gathered}
$$

Proof. Thanks to the consistency condition in $\mathbf{H} 2$ and the continuity of $g$ and $h$, we can extend by Tietze's extension theorem (see [32, Section 2.6]) the functions $g, h$ from the closed set $\{0\} \times \bar{D} \cup[0, \infty) \times \partial D$ to a continuous function $G$ defined in $[0, \infty) \times \mathbb{R}^{d}$.

As a consequence of Proposition 3.2, we can write $v$ in (3.4) as follows:

$$
\begin{aligned}
v(t, x)= & \mathbb{E}_{x}\left[\int_{0}^{t \wedge \tau_{D}} e^{\int_{0}^{s} c(t-r, X(r)) d r} f(t-s, X(s)) d s\right] \\
& +\mathbb{E}_{x}\left[e^{e_{0}^{t \wedge \tau_{D}} c(t-r, X(r)) d r} G\left(t-t \wedge \tau_{D}, X\left(t \wedge \tau_{D}\right)\right)\right] .
\end{aligned}
$$

We are ready to prove both lemmas explained above.

Lemma 3.3. Assume $\mathbf{H 0}, \mathbf{H 1}$, and $\mathbf{H 2}$. Let $v$ be defined as in (3.6) and assume that $v \in C([0, \infty) \times$ $\bar{D}) \cap C^{1,2}((0, \infty) \times D)$. Then, $v$ fulfils the following equation:

$$
\begin{gathered}
-u_{t}(t, x)+\mathcal{L}[u](t, x)+c(t, x) u(t, x)=-f(t, x) \quad(t, x) \in(0, \infty) \times D, \\
u(t, x)=G(t, x) \quad(t, x) \in \partial((0, \infty) \times D) .
\end{gathered}
$$

Furthermore, for all $T>0$, there exists $C$ such that

$$
\sup _{0 \leq t \leq T}|v(t, x)| \leq C\left(T, c_{0}, K_{1}, K_{2}, K_{3}, k\right)\left(1+\|x\|^{k}\right), \quad x \in \bar{D}
$$

where $c_{0}, K_{1}, K_{2}, K_{3}$, and $k$ are the constants defined in $\mathbf{H 1}$ and $\mathbf{H} \mathbf{2}$.

Proof. Let $0 \leq \alpha \leq t$, then, following the same argument used to prove (4.80) in the proof of Theorem 4.4 in Section 4 we have that

$$
\begin{array}{r}
\mathbb{E}_{x}\left[\int_{0}^{t \wedge \tau_{D}} e^{\int_{0}^{s} c(t-r, X(r)) d r} f(t-s, X(s)) d s+e^{\int_{0}^{t \wedge \tau_{D}} c(t-r, X(r)) d r} G\left(t-t \wedge \tau_{D}, X\left(t \wedge \tau_{D}\right)\right) \mid \mathcal{F}_{\alpha}\right] \\
\quad=\int_{0}^{\alpha \wedge \tau_{D}} e^{\int_{0}^{s} c(t-r, X(r)) d r} f(t-s, X(s)) d s+e^{\int_{0}^{\alpha \wedge \tau_{D}} c(t-r, X(r))} v\left(t-\alpha \wedge \tau_{D}, X\left(\alpha \wedge \tau_{D}\right)\right) .
\end{array}
$$


Because of $\mathbf{H 1}$ and $\mathbf{H} \mathbf{2}$, we have that the random variable inside the conditional expectation is integrable and so the left-hand side of (3.9) is a $\mathcal{F}_{\alpha}$-martingale, for $\alpha \in[0, t]$. Since $v \in C^{1,2}$, we can apply Ito's formula to $e^{\int_{0}^{\alpha} c d r} v()$ to get

$$
\begin{aligned}
e^{\int_{0}^{\alpha \wedge \tau_{D}} c(t-r, X(r)) d r} v\left(t-\alpha \wedge \tau_{D}, X\left(\alpha \wedge \tau_{D}\right)\right) \\
=v(t, x)+\int_{0}^{\alpha \wedge \tau_{D}} e^{\int_{0}^{s} c(t-r, X(r)) d r}\left(-v_{t}+\mathcal{L}[v]+c v\right)(t-s, X(s)) d s \\
\quad+\int_{0}^{\alpha \wedge \tau_{D}} D v(t-s, X(s)) \cdot \sigma(t-s, X(s)) d W(s) .
\end{aligned}
$$

It follows from the continuity of $D v, \sigma$ and $X(\cdot)$ that

$$
\sup _{0 \leq s \leq \alpha}\|D v(t-s, X(s))\|\|\sigma(t-s, X(s))\|
$$

is a.s. finite and then

$$
\int_{0}^{\alpha \wedge \tau_{D}} D v(t-s, X(s)) \cdot \sigma(t-s, X(s)) d W(s)
$$

is a local martingale for $0 \leq \alpha \leq t$. So, combining (3.9) and (3.10), we get that

$$
M(\alpha):=\int_{0}^{\alpha \wedge \tau_{D}} e^{\int_{0}^{s} c(t-r, X(r)) d r}\left(-v_{t}+\mathcal{L}[v]+c v+f\right)(t-s, X(s)) d s
$$

is a continuous local martingale for $\alpha \in[0, t]$. Since $M$ is locally of bounded variation, then $M(\alpha) \equiv 0$. This implies that $-v_{t}+\mathcal{L}[v]+c v+f=0$ for all $(t, x) \in(0, \infty) \times D$.

For the boundary condition, it follows from the regularity of the set $D$ and the local ellipticity (see Remark 2.4) that

$$
\mathbb{P}_{x}\left[t \wedge \tau_{D}=0\right]=1, \quad \text { for }(t, x) \in \partial([0, \infty) \times \bar{D})
$$

From this, it is clear that the first addend of the right-hand side of (3.6) is zero. For the second addend, we get that the exponential term is equal to one and that $\mathbb{E}_{x}\left[G\left(t-t \wedge \tau_{D}, X\left(t \wedge \tau_{D}\right)\right)\right]=$ $G(t, X(0))=G(t, x)$, and so we conclude that $v$ satisfies the boundary condition.

The second statement of the theorem is proved with the same argument used to prove (4.9) and (4.42) in the proofs of Lemmas 4.2 and 4.3 in Section 4. 
The next Lemma proves the uniqueness of the solution.

Lemma 3.4. Assume H0, H1, and H2. Assume that there exists a classical solution $u \in C([0, \infty) \times$ $\bar{D}) \cap C^{1,2}((0, \infty) \cap D)$ to equations

$$
\begin{gathered}
-u_{t}(t, x)+\mathcal{L}[u](t, x)+c(t, x) u(t, x)=-f(t, x) \quad(t, x) \in(0, \infty) \times D, \\
u(t, x)=G(t, x) \quad(t, x) \in \partial((0, \infty) \times D),
\end{gathered}
$$

such that, for all $T>0$, there exists $C$ for which

$$
\sup _{0 \leq t \leq T}|u(t, x)| \leq C(T)\left(1+\|x\|^{\mu}\right)
$$

for some $\mu>0$. Then, $u$ has the following representation:

$$
\begin{aligned}
& u(t, x)=\mathbb{E}_{x}\left[\int_{0}^{t \wedge \tau_{D}} e^{\int_{0}^{s} c(t-r, X(r)) d r} f(t-s, X(s)) d s\right] \\
& +\mathbb{E}_{x}\left[e^{\int_{0}^{t \wedge \tau_{D}} c(t-r, X(r)) d r} G\left(t-t \wedge \tau_{D}, X\left(t \wedge \tau_{D}\right)\right)\right],
\end{aligned}
$$

and hence the solution is unique.

Proof. Consider, for $\alpha \in[0, t]$, the process

$$
e^{\int_{0}^{\alpha \wedge \tau_{D}} c(t-r, X(r)) d r} u\left(t-\alpha \wedge \tau_{D}, X\left(\alpha \wedge \tau_{D}\right)\right) .
$$

Applying Ito's rule, we get

$$
\begin{aligned}
e^{\int_{0}^{\alpha \wedge \tau_{D}} c(t-r, X(r)) d r} u\left(t-\alpha \wedge \tau_{D}, X\left(\alpha \wedge \tau_{D}\right)\right) \\
=u(t, x)+\int_{0}^{\alpha \wedge \tau_{D}} e^{\int_{0}^{s} c(t-r, X(r)) d r}\left(-u_{t}+\mathcal{L}[u]+c u\right)(t-s, X(s)) d s \\
\quad+\int_{0}^{\alpha \wedge \tau_{D}} D u(t-s, X(s)) \cdot \sigma(t-s, X(s)) d W(s) .
\end{aligned}
$$

A similar argument as the one used in the proof of Lemma 3.3 shows that

$$
\int_{0}^{\alpha \wedge \tau_{D}} D u(t-s, X(s)) \cdot \sigma(t-s, X(s)) d W(s)
$$

is a local martingale. Due to (3.15), we conclude that

$$
M(\alpha):=e^{\int_{0}^{\alpha \wedge \tau_{D}} c(t-r, X(r)) d r} u\left(t-\alpha \wedge \tau_{D}, X\left(\alpha \wedge \tau_{D}\right)\right)+\int_{0}^{\alpha \wedge \tau_{D}} e^{\int_{0}^{s} c(t-r, X(r)) d r} f(t-s, X(s)) d s
$$


is a local martingale for $\alpha \in[0, t]$. Let $\left\{\theta_{n}\right\}_{n \geq 1}$ be a sequence of localization times for $M(\alpha)$; that is, $\theta_{n} \uparrow \infty$ a.s. as $n \rightarrow \infty$ and $M\left(\alpha \wedge \theta_{n}\right)$ is a martingale for all $n \geq 1$. Then, for all $n \geq 1$,

$$
\begin{aligned}
u(t, x)= & \mathbb{E}_{x}\left[e^{\int_{0}^{\alpha \wedge \tau_{D} \wedge \theta_{n}} c(t-r, X(r)) d r} u\left(t-\alpha \wedge \tau_{D} \wedge \theta_{n}, X\left(\alpha \wedge \tau_{D} \wedge \theta_{n}\right)\right)\right] \\
& +\mathbb{E}_{x}\left[\int_{0}^{\alpha \wedge \tau_{D} \wedge \theta_{n}} e^{\int_{0}^{s} c(t-r, X(r)) d r} f(t-s, X(s)) d s\right] .
\end{aligned}
$$

Since $0 \leq \alpha \wedge \tau_{D} \wedge \theta_{n} \leq t$, using estimate (3.16), we get

$$
\begin{gathered}
e^{\int_{0}^{\alpha \wedge \tau_{D} \wedge \theta_{n}} c(t-r, X(r)) d r}\left|u\left(t-\alpha \wedge \tau_{D} \wedge \theta_{n}, X\left(\alpha \wedge \tau_{D} \wedge \theta_{n}\right)\right)\right| \\
\leq e^{c_{0} t} C(t)\left(1+\left\|X\left(\alpha \wedge \tau_{D} \wedge \theta_{n}\right)\right\|^{\mu}\right) \\
\leq e^{c_{0} t} C(t)\left(1+\sup _{0 \leq s \leq t}\|X(s)\|^{\mu}\right) \\
\left|\int_{0}^{\alpha \wedge \tau_{D} \wedge \theta_{n}} e^{\int_{0}^{s} c(t-r, X(r)) d r} f(t-s, X(s)) d s\right| \\
\leq \int_{0}^{\alpha \wedge \tau_{D} \wedge \theta_{n}} e^{c_{0} s} K_{2}(t)\left(1+\|X(s)\|^{k}\right) d s \\
\leq e^{c_{0} t} t K_{2}(t)\left(1+\sup _{0 \leq s \leq t}\|X(s)\|^{k}\right) .
\end{gathered}
$$

By (2.15) and the dominated convergence theorem, letting $n \rightarrow \infty$, we get

$$
\begin{aligned}
u(t, x)= & \mathbb{E}_{x}\left[e^{\int_{0}^{\alpha \wedge \tau_{D}} c(t-r, X(r)) d r} u\left(t-\alpha \wedge \tau_{D}, X\left(\alpha \wedge \tau_{D}\right)\right)\right] \\
& +\mathbb{E}_{x}\left[\int_{0}^{\alpha \wedge \tau_{D}} e^{\int_{0}^{s} c(t-r, X(r)) d r} f(t-s, X(s)) d s\right] .
\end{aligned}
$$

Letting $\alpha \uparrow t$, a similar argument and the boundary condition proof that

$$
\begin{aligned}
u(t, x)= & \mathbb{E}_{x}\left[e^{\int_{0}^{t \wedge \tau_{D}} c(t-r, X(r)) d r} G\left(t-t \wedge \tau_{D}, X\left(t \wedge \tau_{D}\right)\right)\right] \\
& +\mathbb{E}_{x}\left[\int_{0}^{t \wedge \tau_{D}} e^{\int_{0}^{s} c(t-r, X(r)) d r} f(t-s, X(s)) d s\right],
\end{aligned}
$$

and the proof is complete. 


\section{Regularity of $v$}

In this section, we prove that $v \in C([0, \infty) \times \bar{D}) \cap C_{\text {loc }}^{1,2, \lambda}((0, \infty) \times D)$. First, we prove, using the continuity of the flow process $X$, that $v$ is a continuous function in $[0, \infty) \times \bar{D}$. Since we are only assuming the continuity of the coefficients, then the flow is not necessarily differentiable and so we can not prove the regularity of $v$ in terms of the regularity of the flow. To prove that $v \in C^{1,2}$, we show that $v$ is the solution to a parabolic differential equation in a bounded domain, for which we have the existence of a classical solution and hence $v \in C^{1,2}$.

\subsection{Continuity of $v$}

Let $(\xi, X)$ denote the solution to (2.3) and let $G$ be defined as in Proposition 3.2, then $v$ has the following form:

$$
\begin{aligned}
v(t, x)= & \mathbb{E}_{t, x}\left[\int_{0}^{t \wedge \tau_{D}} e^{\int_{0}^{s} c(\xi(r), X(r)) d r} f(\xi(s), X(s)) d s\right] \\
& +\mathbb{E}_{t, x}\left[e^{\int_{0}^{t \wedge \tau_{D}} c(\xi(r), X(r)) d r} G\left(\xi\left(t \wedge \tau_{D}\right), X\left(t \wedge \tau_{D}\right)\right)\right] .
\end{aligned}
$$

For simplicity, we write $v=v_{1}+v_{2}$, where

$$
\begin{aligned}
& v_{1}(t, x):=\mathbb{E}_{t, x}\left[\int_{0}^{t \wedge \tau_{D}} e^{\int_{0}^{s} c(\xi(r), X(r)) d r} f(\xi(s), X(s)) d s\right], \\
& v_{2}(t, x):=\mathbb{E}_{t, x}\left[e^{e_{0}^{t \wedge \tau_{D}} c(\xi(r), X(r)) d r} G\left(\xi\left(t \wedge \tau_{D}\right), X\left(t \wedge \tau_{D}\right)\right)\right] .
\end{aligned}
$$

Theorem 4.1. Assume H0, H1, and H2. Let $v$ be defined as in (4.1). Then, $v$ is continuous on $[0, \infty) \times \bar{D}$.

The proof of this theorem is divided into two lemmas.

Lemma 4.2. Assume H0, H1, and $\mathbf{H 2}$. Let $v_{1}$ be defined as in (4.2). Then, $v_{1}$ is continuous on $[0, \infty) \times \bar{D}$.

Proof. First, we prove the continuity on $(0, \infty) \times D$. For that, let

$$
\left(t_{n}, x_{n}\right) \underset{n \rightarrow \infty}{\longrightarrow}(t, x)
$$

in $(0, \infty) \times D$ and $\epsilon>0$. We need to prove that there exists $N(\epsilon) \in \mathbb{N}$ such that, for all $n \geq N(\epsilon)$

$$
\left|v_{1}\left(t_{n}, x_{n}\right)-v_{1}(t, x)\right|<\epsilon .
$$

Denote by $(\xi, X)$ and $\left(\xi_{n}, X_{n}\right)$ the solutions to (2.3) with initial conditions $(t, x)$ and $\left(t_{n}, x_{n}\right)$, respectively. To simplify the notation in the proof, let $\tau:=t \wedge \tau_{D}$ and $\tau_{n}:=t_{n} \wedge \tau_{D n}$ denote their corresponding exit times from $[0, \infty) \times \bar{D}$. 
Let $\alpha>0$, then there exists $N_{1} \in \mathbb{N}$ such that, for all $n \geq N_{1}$

$$
\left\|\left(t_{n}, x_{n}\right)-(t, x)\right\|<\alpha .
$$

Observe that, for all $n \geq N_{1}$, we get

$$
\begin{gathered}
\tau_{n} \leq t_{n} \leq t+\alpha, \\
\tau \leq t \leq t+\alpha .
\end{gathered}
$$

Define the random variables $Y_{n}$ as

$$
Y_{n}:=\left|\int_{0}^{\tau_{n}} e^{\int_{0}^{S} c\left(\xi_{n}(r), X_{n}(r)\right) d r} f\left(\xi_{n}(s), X_{n}(s)\right) d s-\int_{0}^{\tau} e^{\int_{0}^{s} c(\xi(r), X(r)) d r} f(\xi(s), X(s)) d s\right| .
$$

The sequence $\left\{Y_{n}\right\}_{n \geq N_{1}}$ is uniformly integrable. Thanks to Theorem 4.2, in Chapter 5 of [33], it is sufficient to prove that $\sup _{n} \mathbb{E}\left[Y_{n}^{2}\right]<\infty$. So,

$$
\begin{aligned}
\mathbb{E}\left[Y_{n}^{2}\right] \leq & 2 \mathbb{E}\left[\left|\int_{0}^{\tau_{n}} e^{\int_{0}^{s} c\left(\xi_{n}(r), X_{n}(r)\right) d r} f\left(\xi_{n}(s), X_{n}(s)\right) d s\right|^{2}\right] \\
& +2 \mathbb{E}\left[\left|\int_{0}^{\tau} e^{\int_{0}^{s} c(\xi(r), X(r)) d r} f(\xi(s), X(s)) d s\right|^{2}\right] \\
\leq & 2 \mathbb{E}\left[\left|\int_{0}^{\tau_{n}} e^{c_{0}(t+\alpha)} K_{2}(t+\alpha)\left(1+\left\|X_{n}(s)\right\|^{k}\right) d s\right|^{2}\right] \\
& +2 \mathbb{E}\left[\left|\int_{0}^{\tau} e^{c_{0}(t+\alpha)} K_{2}(t+\alpha)\left(1+\|X(s)\|^{k}\right) d s\right|^{2}\right] \\
\leq & 2 e^{2 c_{0}(t+\alpha)} K_{2}^{2}(t+\alpha) \mathbb{E}\left[\left|\int_{0}^{\tau_{n}}\left(1+\sup _{0 \leq r \leq t+\alpha}\left\|X_{n}(r)\right\|^{k}\right) d s\right|^{2}\right] \\
& +2 e^{2 c_{0}(t+\alpha)} K_{2}^{2}(t+\alpha) \mathbb{E}\left[\left|\int_{0}^{\tau}\left(1+\sup _{0 \leq r \leq t+\alpha}\|X(r)\|^{k}\right) d s\right|^{2}\right] \\
\leq & 4 e^{2 c_{0}(t+\alpha)} K_{2}^{2}(t+\alpha)(t+\alpha)^{2}\left(1+\mathbb{E}\left[\sup _{0 \leq r \leq t+\alpha}\left\|X_{n}(r)\right\|^{2 k}\right]\right) \\
& +4 e^{2 c_{0}(t+\alpha)} K_{2}^{2}(t+\alpha)(t+\alpha)^{2}\left(1+\mathbb{E}\left[\sup _{0 \leq r \leq t+\alpha}\|X(r)\|^{2 k}\right]\right)
\end{aligned}
$$




$$
\begin{aligned}
& \leq C\left(1+K\left(1+\left\|x_{n}\right\|^{2 k}\right)\right)+C\left(1+K\left(1+\|x\|^{2 k}\right)\right) \\
& \leq C\left(1+K\left(1+(\|x\|+\alpha)^{2 k}\right)\right)+C\left(1+K\left(1+\|x\|^{2 k}\right)\right)<\infty,
\end{aligned}
$$

where $C=C\left(t, \alpha, c_{0}\right)$ and $K=K(t, \alpha, k)$. We use (4.6), (4.7), (2.15) and the polynomial growth of $f$.

Let $M>0,0<\eta<1$, and $\beta>0$ and define the set

$$
E_{M, n, \eta, \beta}:=\left\{\|X\|_{t+\alpha} \leq M\right\} \cap\left\{\left\|X_{n}-X\right\|_{t+\alpha} \leq \eta\right\} \cap\left\{\left|\tau_{n}-\tau\right| \leq \beta\right\} .
$$

Then,

$$
\left|v_{1}\left(t_{n}, x_{n}\right)-v_{1}(t, x)\right| \leq \int_{\Omega} \Upsilon_{n} d \mathbb{P}=\int_{E_{M, n, \eta, \beta}} \Upsilon_{n} d \mathbb{P}+\int_{\Omega \backslash E_{M, n, n, \beta}} \Upsilon_{n} d \mathbb{P}
$$

Since the sequence $\left\{Y_{n}\right\}$ is uniformly integrable, there exists $\delta(\epsilon)$ such that for any $E \in \mathcal{F}$ that satisfies $\mathbb{P}[E]<\delta$, we have

$$
\sup _{n \geq N_{1}} \int_{E} Y_{n} d \mathbb{P}<\frac{\epsilon}{2}
$$

It follows, from Remark 2.4, Proposition 2.5, and Theorems 5.3 and 5.4 in Section 5, the existence of $M$ and $N_{2}$ such that

$$
\mathbb{P}\left[\Omega \backslash E_{M, n, \eta, \beta}\right]<\delta(\epsilon)
$$

for all $n \geq N_{2}$. Then for $n \geq N_{1} \vee N_{2}$, we get that

$$
\left|v_{1}\left(t_{n}, x_{n}\right)-v_{1}(t, x)\right| \leq \int_{E_{M, n, \eta, \beta}} \Upsilon_{n} d \mathbb{P}+\frac{\epsilon}{2}
$$

For simplicity of notation, we write the set $E_{M, n, \eta, \beta}$ as $E$ and define

$$
\begin{gathered}
A:=[0, t+\alpha] \times[-M-1, M+1]^{d}, \\
D_{t}:=[0, t+\alpha] \times \bar{D}
\end{gathered}
$$

and let

$$
B_{A}:=(-m, m)^{d+1}
$$

be an open set such that $A \subset B_{A}$. 
International Journal of Stochastic Analysis

On the set $E$, for all $n \geq N_{1}$ and $0 \leq s \leq t+\alpha$, it is satisfied that

$$
\left(\xi_{n}(s), X_{n}(s)\right),(\xi(s), X(s)) \in A .
$$

We have that

$$
\begin{aligned}
\int_{E} Y_{n} d \mathbb{P} \leq & \int_{E} \int_{0}^{\tau_{n} \wedge \tau}\left|e^{\int_{0}^{s} c\left(\xi_{n}(r), X_{n}(r)\right) d r} f\left(\xi_{n}(s), X_{n}(s)\right)-e^{\int_{0}^{s} c(\xi(r), X(r)) d r} f(\xi(s), X(s))\right| d s d \mathbb{P} \\
& +\int_{E} \int_{\tau_{n} \wedge \tau}^{\tau_{n} \vee \tau}\left(e^{\int_{0}^{s} c\left(\xi_{n}(r), X_{n}(r)\right) d r}\left|f\left(\xi_{n}(s), X_{n}(s)\right)\right| \mathbb{1}_{\tau_{n}>\tau}\right. \\
& \left.+e^{\int_{0}^{s} c(\xi(r), X(r)) d r}|f(\xi(s), X(s))| \mathbb{1}_{\tau_{n} \leq \tau}\right) d s d \mathbb{P} .
\end{aligned}
$$

We first analyse (4.19):

$$
\begin{aligned}
(4.19) & \leq \int_{E} \int_{\tau_{n} \wedge \tau}^{\tau_{n} \vee \tau} e^{c_{0}(t+\alpha)} K_{f}\left(A \cap D_{t}\right)\left(\mathbb{1}_{\tau_{n} \leq \tau}+\mathbb{1}_{\tau_{n}>\tau}\right) d s d \mathbb{P} \\
& =e^{c_{0}(t+\alpha)} K_{f}\left(A \cap D_{t}\right) \int_{E}\left|\tau_{n}-\tau\right| d \mathbb{P} \\
& \leq e^{c_{0}(t+\alpha)} K_{f}\left(A \cap D_{t}\right) \beta .
\end{aligned}
$$

For (4.18), we get

$$
\begin{aligned}
(4.18) \leq & \int_{E} \int_{0}^{\tau_{n} \wedge \tau} e^{\int_{0}^{s} c\left(\xi_{n}(r), X_{n}(r)\right) d r}\left|f\left(\xi_{n}(s), X_{n}(s)\right)-f(\xi(s), X(s))\right| d s d \mathbb{P} \\
& +\int_{E} \int_{0}^{\tau_{n} \wedge \tau}|f(\xi(s), X(s))|\left|e^{\int_{0}^{s} c\left(\xi_{n}(r), X_{n}(r)\right) d r}-e^{\int_{0}^{s} c(\xi(r), X(r)) d r}\right| d s d \mathbb{P} .
\end{aligned}
$$

Now,

$$
\begin{aligned}
(4.21) & \leq \int_{E} e^{c_{0}(t+\alpha)} \int_{0}^{\tau_{n} \wedge \tau} L_{f}\left(B_{A}\right)\left(\left|t_{n}-t\right|^{\lambda}+\left\|X_{n}(s)-X(s)\right\|\right) d s d \mathbb{P} \\
& \leq e^{c_{0}(t+\alpha)}(t+\alpha) L_{f}\left(B_{A}\right)\left(\left|t_{n}-t\right|^{\lambda}+\eta\right) .
\end{aligned}
$$


For (4.22), we need the following bound:

$$
\begin{aligned}
& \left|e^{\int_{0}^{s} c\left(\xi_{n}(r), X_{n}(r)\right) d r}-e^{\int_{0}^{s} c(\xi(r), X(r)) d r}\right| \\
& \quad=e^{\int_{0}^{s} c(\xi(r), X(r)) d r}\left|\exp \left\{\int_{0}^{s}\left(c\left(\xi_{n}(r), X_{n}(r)\right)-c(\xi(r), X(r))\right) d r\right\}-1\right| \\
& \quad \leq e^{c_{0} s}\left(\exp \left\{\int_{0}^{s}\left|c\left(\xi_{n}(r), X_{n}(r)\right)-c(\xi(r), X(r))\right| d r\right\}-1\right) \\
& \quad \leq e^{c_{0} s}\left(\exp \left\{\int_{0}^{s} L_{c}\left(B_{A}\right)\left(\left|t_{n}-t\right|^{\lambda}+\left\|X_{n}(r)-X(r)\right\|\right) d r\right\}-1\right) \\
& \quad \leq e^{c_{0} s}\left(\exp \left\{L_{c}\left(B_{A}\right) s\left(\left|t_{n}-t\right|^{\lambda}+\eta\right)\right\}-1\right),
\end{aligned}
$$

since $\left|e^{x}-1\right| \leq e^{|x|}-1$. If we choose $N_{3} \in \mathbb{N}$ such that $\left|t_{n}-t\right|^{\lambda} \leq 1 /\left(2 L_{c}\left(B_{A}\right)(t+\alpha)\right)$ for all $n \geq N_{3}$ and $\eta \leq 1 /\left(2 L_{c}\left(B_{A}\right)(t+\alpha)\right)$, then we get by the mean value theorem that

$$
\left|e^{\int_{0}^{s} c\left(\xi_{n}(r), X_{n}(r)\right) d r}-e^{\int_{0}^{s} c(\xi(r), X(r)) d r}\right| \leq e^{c_{0} s} e L_{c}\left(B_{A}\right) s\left(\left|t_{n}-t\right|^{\lambda}+\eta\right) .
$$

Then,

$$
(4.22) \leq K_{f}\left(A \cap D_{t}\right) e^{c_{0}(t+\alpha)} e L_{c}\left(B_{A}\right)(t+\alpha)^{2}\left(\left|t_{n}-t\right|^{\lambda}+\eta\right) .
$$

To summarize, we get with the above estimations that

$$
\begin{aligned}
\int_{E} Y_{n} d \mathbb{P} \leq & e^{c_{0}(t+\alpha)}(t+\alpha) L_{f}\left(B_{A}\right)\left(\left|t_{n}-t\right|^{\lambda}+\eta\right) \\
& +K_{f}\left(A \cap D_{t}\right) e^{c_{0}(t+\alpha)} e L_{c}\left(B_{A}\right)(t+\alpha)^{2}\left(\left|t_{n}-t\right|^{\lambda}+\eta\right) \\
& +e^{c_{0}(t+\alpha)} K_{f}\left(A \cap D_{t}\right) \beta .
\end{aligned}
$$

Hence, to prove continuity, we proceed as follows,

(i) Let $\epsilon>0$ and $0<\alpha \ll 1$.

(ii) Let $N_{1} \geq \mathbb{N}$ such that, for all $n \geq N_{1}$,

$$
\left\|\left(t_{n}, x_{n}\right)-(t, x)\right\|<\alpha .
$$

(iii) Let $\delta(\epsilon)>0$ fulfil the uniformly integrability condition (4.12).

(iv) Take $M>0$ such that $\mathbb{P}\left[\|X\|_{t+\alpha}>M\right]<\delta(\epsilon) / 3$.

(v) Define $A:=[0, t+\alpha] \times[-M-1, M+1]^{d}$ and $D_{t}:=[0, t+\alpha] \times \bar{D}$. 
(vi) Let

$$
\eta<\min \left\{1, \frac{1}{2 L_{c}\left(B_{A}\right)(t+\alpha)}, \frac{\epsilon}{16 e^{c_{0}(t+\alpha)}(t+\alpha) L_{f}\left(B_{A}\right)}, \frac{\epsilon}{16 K_{f}\left(A \cap D_{t}\right) e^{c_{0}(t+\alpha)} e L_{c}\left(B_{A}\right)(t+\alpha)^{2}}\right\}
$$

(vii) Choose $N_{2} \in \mathbb{N}$ such that $\mathbb{P}\left[\left\|X_{n}-X\right\|_{t+\alpha}>\eta\right] \leq \delta(\epsilon) / 3$ for all $n \geq N_{2}$

(viii) Let $N_{3} \in \mathbb{N}$ be such that

$$
\left|t_{n}-t\right|^{\lambda}<\min \left\{\frac{1}{2 L_{c}\left(B_{A}\right)(t+\alpha)}, \frac{\epsilon}{16 e^{c_{0}(t+\alpha)}(t+\alpha) L_{f}\left(B_{A}\right)}, \frac{\epsilon}{16 K_{f}\left(A \cap D_{t}\right) e^{c_{0}(t+\alpha)} e L_{c}\left(B_{A}\right)(t+\alpha)}\right\}
$$

for all $n \geq N_{3}$.

(ix) Let

$$
\beta<\frac{\epsilon}{4 e^{c_{0}(t+\alpha)} K_{f}\left(A \cap D_{t}\right)}
$$

and choose $N_{4} \in \mathbb{N}$ such that, for all $n \geq N_{4}, \mathbb{P}\left[\left|\tau_{n}-\tau\right|>\beta\right] \leq \delta(\epsilon) / 3$.

Thus, if $N=N_{1} \vee N_{2} \vee N_{3} \vee N_{4}$, then, for all $n \geq N$,

$$
\left|v_{1}\left(t_{n}, x_{n}\right)-v_{1}(t, x)\right|<\epsilon .
$$

Therefore, $v_{1}$ is continuous in $(0, \infty) \times D$.

For the continuity at the boundary we make a similar argument. Let $\left(t_{n}, x_{n}\right) \underset{n \rightarrow \infty}{\rightarrow}(t, x)$, where $\left(t_{n}, x_{n}\right) \in(0, \infty) \times D$ and $(t, x) \in \partial((0, \infty) \times D)$, that is, either $t=0$ or $x \in \partial D$. In both cases we get that $\tau=0$ a.s. and so $v_{1}(t, x)=0$. Then, we need to prove that

$$
\left|v_{1}\left(t_{n}, x_{n}\right)\right| \underset{n \rightarrow \infty}{\longrightarrow} 0 .
$$

Let $0<\alpha \ll 1$ and $N_{1} \in \mathbb{N}$ be such that

$$
\left\|\left(t_{n}, x_{n}\right)-(t, x)\right\|<\alpha .
$$

We get

$$
\tau_{n} \leq t_{n}<t+\alpha
$$


for all $n \geq N_{1}$. For the continuity, we have

$$
\begin{aligned}
\left|v_{1}\left(t_{n}, x_{n}\right)\right| & \leq \mathbb{E}\left[\int_{0}^{\tau_{n}} e^{\int_{0}^{s} c\left(\xi_{n}(r), X_{n}(r)\right) d r}\left|f\left(\xi_{n}(s), X_{n}(s)\right)\right| d s\right] \\
& \leq \mathbb{E}\left[\int_{0}^{\tau_{n}} e^{c_{0} s} K_{2}(t+\alpha)\left(1+\left\|X_{n}(s)\right\|^{k}\right) d s\right] \\
& \leq e^{c_{0}(t+\alpha)} K_{2}(t+\alpha) \mathbb{E}\left[\tau_{n}\left(1+\sup _{0 \leq r \leq t+\alpha}\left\|X_{n}(r)\right\|^{k}\right)\right] \underset{n \rightarrow \infty}{\longrightarrow} 0 .
\end{aligned}
$$

The convergence follows from the uniform integrability of

$$
\tau_{n}\left(1+\sup _{0 \leq r \leq t+\alpha}\left\|X_{n}(r)\right\|^{k}\right)
$$

and the fact that $\tau_{n} \underset{n \rightarrow \infty}{\stackrel{\mathbb{P}}{\rightarrow}} 0$ (see Theorem 5.2 in Chapter 5 of [33] and Theorem 5.3 in Section 5). This completes the proof.

Lemma 4.3. Assume H0, H1, and H2. Let $\boldsymbol{v}_{2}$ be defined as in (4.3). Then, $v_{2}$ is continuous on $[0, \infty) \times \bar{D}$.

Proof. We use an analogous argument to the one in the proof of Lemma 4.2. First, we prove the continuity in $(0, \infty) \times D$. Let

$$
\left(t_{n}, x_{n}\right) \underset{n \rightarrow \infty}{\longrightarrow}(t, x)
$$

with $\left(t_{n}, x_{n}\right),(t, x) \in(0, \infty) \times D$. Denote by $\left(\xi_{n}, X_{n}\right)$ and $(\xi, X)$ the solutions to (2.3) with initial conditions $\left(t_{n}, x_{n}\right)$ and $(t, x)$, respectively, and let $\tau_{n}:=t_{n} \wedge \tau_{D n}$ and $\tau:=t \wedge \tau_{D}$ be their corresponding exit times from $[0, \infty) \times \bar{D}$. Let $0<\alpha \ll 1$ and $N_{1}$ be such that, for all $n \geq N_{1}$,

$$
\left\|\left(t_{n}, x_{n}\right)-(t, x)\right\|<\alpha
$$

This implies that

$$
\begin{gathered}
\tau_{n} \leq t+\alpha, \\
\tau \leq t+\alpha .
\end{gathered}
$$

First, we prove that the sequence of random variables

$$
Y_{n}:=\left|e^{\int_{0}^{\tau_{n}} c\left(\xi(\xi), X_{n}(r)\right) d r} G\left(\xi_{n}\left(\tau_{n}\right), X_{n}\left(\tau_{n}\right)\right)-e^{\int_{0}^{\tau} c(\xi(r), X(r)) d r} G(\xi(\tau), X(\tau))\right|
$$


is uniformly integrable for all $n \geq N_{1}$. As in (4.9),

$$
\begin{aligned}
& \mathbb{E}\left[Y_{n}^{2}\right] \leq 2 \mathbb{E}\left[\left|e^{\tau_{0}^{\tau_{n}} c\left(\xi_{n}(r), X_{n}(r)\right) d r} G\left(\xi_{n}\left(\tau_{n}\right), X_{n}\left(\tau_{n}\right)\right)\right|^{2}\right] \\
& +2 \mathbb{E}\left[\left|e^{\int_{0}^{\tau} c(\xi(r), X(r)) d r} G(\xi(\tau), X(\tau))\right|^{2}\right] \\
& \leq 2 \mathbb{E}\left[e^{2 c_{0}(t+\alpha)} K_{3}^{2}(t+\alpha)\left(1+\left\|X_{n}\left(\tau_{n}\right)\right\|^{k}\right)^{2}\right] \\
& +2 \mathbb{E}\left[e^{2 c_{0}(t+\alpha)} K_{3}^{2}(t+\alpha)\left(1+\|X(\tau)\|^{k}\right)^{2}\right] \\
& \leq 2 e^{2 c_{0}(t+\alpha)} K_{3}^{2}(t+\alpha) \mathbb{E}\left[\left(1+\sup _{0 \leq r \leq t+\alpha}\left\|X_{n}(r)\right\|^{k}\right)^{2}\right] \\
& +2 e^{2 c_{0}(t+\alpha)} K_{3}^{2}(t+\alpha) \mathbb{E}\left[\left(1+\sup _{0 \leq r \leq t+\alpha}\|X(r)\|^{k}\right)^{2}\right] \\
& \leq 4 e^{2 c_{0}(t+\alpha)} K_{3}^{2}(t+\alpha)\left(1+\mathbb{E}\left[\sup _{0 \leq r \leq t+\alpha}\left\|X_{n}(r)\right\|^{2 k}\right]\right) \\
& +4 e^{2 c_{0}(t+\alpha)} K_{3}^{2}(t+\alpha)\left(1+\mathbb{E}\left[\sup _{0 \leq r \leq t+\alpha}\|X(r)\|^{2 k}\right]\right) \\
& \leq C\left(1+K\left(1+\left\|x_{n}\right\|^{2 k}\right)\right)+C\left(1+K\left(1+\|x\|^{2 k}\right)\right) \\
& \leq C\left(1+K\left(1+(\|x\|+\alpha)^{2 k}\right)\right)+C\left(1+K\left(1+\|x\|^{2 k}\right)\right)<\infty,
\end{aligned}
$$

where $C=C\left(t, \alpha, c_{0}\right)$ and $K=K(t, \alpha, k)$. We use (4.39), (4.40), (2.15), and the polynomial growth of $G$ in $\partial((0, \infty) \times D)$. As in Lemma 4.2, let $\epsilon>0$, then there exists $\delta(\epsilon)>0$ such that

$$
\sup _{n \geq N_{1}} \int_{E} Y_{n} d \mathbb{P}<\frac{\epsilon}{2}
$$

for all $E \in \mathcal{F}$, with $\mathbb{P}[E]<\delta(\epsilon)$.

Let $E_{M, n, \eta, \beta}$ be defined as in (4.10), and choose $M>0$ and $N_{2} \in \mathbb{N}$ such that

$$
\mathbb{P}\left[\Omega \backslash E_{M, n, \eta, \beta}\right]<\delta(\epsilon),
$$

for all $n \geq N_{2}$. 
For simplicity of notation, denote $E_{M, n, \eta, \beta}$ as $E$. Then,

$$
\begin{aligned}
\left|v_{2}\left(t_{n}, x_{n}\right)-v_{2}(t, x)\right| & \leq \int_{E} \Upsilon_{n} d \mathbb{P}+\int_{\Omega \backslash E} \Upsilon_{n} d \mathbb{P} \\
& \leq \int_{E} \Upsilon_{n} d \mathbb{P}+\frac{\epsilon}{2} .
\end{aligned}
$$

Let $A, D_{t}$, and $B_{A}$ be defined as in Lemma 4.2 (see (4.15) and (4.16)). Then, on the set $E$, we get that, for all $n \geq N_{1}$ and $0 \leq s \leq t+\alpha$,

$$
\left(\xi_{n}(s), X_{n}(s)\right),(\xi(s), X(s)) \in A .
$$

So,

$$
\begin{aligned}
\int_{E} Y_{n} d \mathbb{P} \leq & \int_{E} e^{\int_{0}^{\tau_{n}} c\left(\xi_{n}(r), X_{n}(r)\right) d r} \times\left|G\left(\xi_{n}\left(\tau_{n}\right), X_{n}\left(\tau_{n}\right)\right)-G(\xi(\tau), X(\tau))\right| d \mathbb{P} \\
& +\int_{E}|G(\xi(\tau), X(\tau))|\left|e^{\int_{0}^{\tau_{n}} c\left(\xi_{n}(r), X_{n}(r)\right) d r}-e^{\int_{0}^{\tau} c(\xi(r), X(r)) d r}\right| d \mathbb{P} .
\end{aligned}
$$

We study each addend of the right-hand side separately:

$$
\begin{aligned}
(4.47) \leq & e^{c_{0}(t+\alpha)} \int_{E}\left|G\left(t_{n}-\tau_{n}, X_{n}\left(\tau_{n}\right)\right)-G\left(t_{n}-\tau_{n}, X\left(\tau_{n}\right)\right)\right| d \mathbb{P} \\
& +e^{c_{0}(t+\alpha)} \int_{E}\left|G\left(t_{n}-\tau_{n}, X\left(\tau_{n}\right)\right)-G(t-\tau, X(\tau))\right| d \mathbb{P} .
\end{aligned}
$$

First, we get a bound for (4.49). Since $G$ is continuous, then it is uniformly continuous on $A$. Then, for $\epsilon>0$, there exists $\gamma\left(c_{0}, t, \alpha, \epsilon, M\right)$ such that

$$
\left|G\left(t_{1}, x_{1}\right)-G\left(t_{2}, x_{2}\right)\right|<\frac{\epsilon}{8 e^{c_{0}(t+\alpha)}}
$$

for all $\left(t_{1}, x_{1}\right),\left(t_{2}, x_{2}\right) \in A$ with $\left\|\left(t_{1}, x_{1}\right)-\left(t_{2}, x_{2}\right)\right\|<\gamma\left(c_{0}, t, \alpha, \epsilon, M\right)$. On the set $E$, we have $\left(t_{n}-\tau_{n}, X_{n}\left(\tau_{n}\right)\right),\left(t_{n}-\tau_{n}, X\left(\tau_{n}\right)\right) \in A$ and

$$
\left\|\left(t_{n}-\tau_{n}, X_{n}\left(\tau_{n}\right)\right)-\left(t_{n}-\tau_{n}, X\left(\tau_{n}\right)\right)\right\|<\eta
$$

So, if we choose $\eta<\gamma$, then we get

$$
(4.49)<\frac{\epsilon}{8} \text {. }
$$


International Journal of Stochastic Analysis

Next, we study (4.50). Thanks to Theorem 5.3, we know that $\tau_{n} \underset{n \rightarrow \infty}{\stackrel{a . s}{\rightarrow}} \tau$. This and the continuity of $X(\cdot)$ and $G$ imply that

$$
G\left(t_{n}-\tau_{n}, X\left(\tau_{n}\right)\right) \underset{n \rightarrow \infty}{\stackrel{a . s .}{\rightarrow}} G(t-\tau, X(\tau))
$$

On the set $E$, we have that $\left(t_{n}-\tau_{n}, X\left(\tau_{n}\right)\right),(t-\tau, X(\tau)) \in A$ and so

$$
\left|G\left(t_{n}-\tau_{n}, X\left(\tau_{n}\right)\right)-G(t-\tau, X(\tau))\right| \mathbb{1}_{E} \leq 2 K_{G}(A) .
$$

By the dominated convergence theorem, there exists $N_{3} \in \mathbb{N}$ such that

$$
(4.50)<\frac{\epsilon}{8}
$$

for all $n \geq N_{3}$.

To give a bound for (4.48), we observe that, on the set $E$,

$$
\begin{aligned}
& \left|\int_{0}^{\tau_{n}} c\left(\xi_{n}(r), X_{n}(r)\right) d r-\int_{0}^{\tau} c(\xi(r), X(r)) d r\right| \\
& \quad \leq \int_{0}^{\tau_{n} \wedge \tau}\left|c\left(\xi_{n}(r), X_{n}(r)\right)-c(\xi(r), X(r))\right| d r \\
& \quad+\int_{\tau_{n} \wedge \tau}^{\tau_{n} \vee \tau}\left(\left|c\left(\xi_{n}(r), X_{n}(r)\right)\right| \mathbb{1}_{\tau_{n} \geq \tau}+|c(\xi(r), X(r))| \mathbb{1}_{\tau_{n}<\tau}\right) d r \\
& \leq \int_{0}^{\tau_{n} \wedge \tau} L_{c}\left(B_{A}\right)\left(\left|t_{n}-t\right|^{\lambda}+\left\|X_{n}(r)-X(r)\right\|\right) d r+K_{c}\left(A \cap D_{t}\right)\left|\tau_{n}-\tau\right| \\
& \leq L_{c}\left(B_{A}\right)(t+\alpha)\left(\left|t_{n}-t\right|^{\lambda}+\eta\right)+K_{c}\left(A \cap D_{t}\right) \beta .
\end{aligned}
$$

Making a similar argument as the one made in (4.24) and (4.25), we get

$$
\begin{aligned}
& \left|e^{\int_{0}^{\tau_{n}} c\left(\xi_{n}(r), X_{n}(r)\right) d r}-e^{\int_{0}^{\tau} c(\xi(r), X(r)) d r}\right| \\
& \quad \leq e^{c_{0}(t+\alpha)} e\left[L_{c}\left(B_{A}\right)(t+\alpha)\left(\left|t_{n}-t\right|^{\lambda}+\eta\right)+K_{c}\left(A \cap D_{t}\right) \beta\right]
\end{aligned}
$$

if $\left|t_{n}-t\right|^{\lambda}<1 / 3 L_{c}\left(B_{A}\right)(t+\alpha), \eta<1 / 3 L_{c}\left(B_{A}\right)(t+\alpha)$, and $\beta<1 / 3 K_{c}\left(A \cap D_{t}\right)$. Then,

$$
(4.48) \leq K_{G}(A) e^{c_{0}(t+\alpha)} e\left[L_{c}\left(B_{A}\right)(t+\alpha)\left(\left|t_{n}-t\right|^{\lambda}+\eta\right)+K_{c}\left(A \cap D_{t}\right) \beta\right] .
$$

To summarize, we get with the above estimations that

$$
\int_{E} Y_{n} d \mathbb{P} \leq \frac{\epsilon}{4}+K_{G}(A) e^{c_{0}(t+\alpha)} e\left[L_{c}\left(B_{A}\right)(t+\alpha)\left(\left|t_{n}-t\right|^{\lambda}+\eta\right)+K_{c}\left(A \cap D_{t}\right) \beta\right] .
$$


Hence, to prove continuity, we proceed as follows.

(i) Let $\epsilon>0$ and $0<\alpha \ll 1$.

(ii) Let $N_{1} \in \mathbb{N}$ be such that, for all $n \geq N_{1}$,

$$
\left\|\left(t_{n}, x_{n}\right)-(t, x)\right\|<\alpha .
$$

(iii) Let $\delta(\epsilon)>0$ fulfil the uniformly integrability condition (4.43).

(iv) Take $M>0$ such that $\mathbb{P}\left[\|X\|_{t+\alpha}>M\right]<\delta(\epsilon) / 3$.

(v) Define $A:=[0, t+\alpha] \times[-M-1, M+1]^{d}$ and $D_{t}:=[0, t+\alpha] \times \bar{D}$.

(vi) Let

$$
\eta<\min \left\{1, \gamma\left(c_{0}, t, \alpha, \epsilon, M\right), \frac{1}{3 L_{c}\left(B_{A}\right)(t+\alpha)}, \frac{\epsilon}{12 K_{G}(A) e^{c_{0}(t+\alpha)} e L_{c}\left(B_{A}\right)(t+\alpha)}\right\}
$$

(vii) Choose $N_{2} \in \mathbb{N}$ such that $\mathbb{P}\left[\left\|X_{n}-X\right\|_{t+\alpha}>\eta\right]<\delta(\epsilon) / 3$ for all $n \geq N_{2}$

(viii) Let

$$
\beta<\min \left\{\frac{1}{3 K_{c}\left(A \cap D_{t}\right)}, \frac{\epsilon}{12 K_{G}(A) e^{c_{0}(t+\alpha)} e K_{c}\left(A \cap D_{t}\right)}\right\} .
$$

(ix) Choose $N_{3} \in \mathbb{N}$ such that $\mathbb{P}\left[\left|\tau_{n}-\tau\right|>\beta\right]<\delta(\epsilon) / 3$ for all $n \geq N_{3}$.

(x) Let $N_{4} \in \mathbb{N}$ be such that

$$
\left|t_{n}-t\right|^{\lambda}<\min \left\{\frac{1}{3 L_{c}\left(B_{A}\right)(t+\alpha)}, \frac{\epsilon}{12 K_{G}(A) e^{c_{0}(t+\alpha)} e L_{c}\left(B_{A}\right)(t+\alpha)}\right\}
$$

for all $n \geq N_{4}$.

(xi) Let $N_{5} \in \mathbb{N}$ to get

$$
\int_{E}\left|G\left(t_{n}-\tau_{n}, X\left(\tau_{n}\right)\right)-G(t-\tau, X(\tau))\right| d \mathbb{P}<\frac{\epsilon}{8 e^{c_{0}(t+\alpha)}}
$$

for all $n \geq N_{5}$.

So, for $N=N_{1} \vee N_{2} \vee N_{3} \vee N_{4} \vee N_{5}$, we have that, if $n \geq N$, then

$$
\left|v_{2}\left(t_{n}, x_{n}\right)-v_{2}(t, x)\right|<\epsilon
$$

and we conclude that $v_{2}$ is continuous over $(0, \infty) \times D$. 
Next, we prove the continuity in the boundary. Let $\left(t_{n}, x_{n}\right) \underset{n \rightarrow \infty}{\rightarrow}(t, x)$, where $\left(t_{n}, x_{n}\right) \in(0, \infty) \times$ $D$ and $(t, x) \in \partial((0, \infty) \times D)$, that is, either $t=0$ or $x \in \partial D$. In both cases, we get that $\tau=0$ a.s., We need to prove that

$$
\left|v_{2}\left(t_{n}, x_{n}\right)-G(t, x)\right| \underset{n \rightarrow \infty}{\longrightarrow} 0 .
$$

Let $0<\alpha \ll 1$ and $N_{1} \in \mathbb{N}$ such that

$$
\left\|\left(t_{n}, x_{n}\right)-(t, x)\right\|<\alpha
$$

So, for all $n \geq N_{1}$,

$$
\tau_{n} \leq t_{n}<t+\alpha
$$

We have that

$$
\begin{aligned}
\left|v_{2}\left(t_{n}, x_{n}\right)-G(t, x)\right| \leq & \mathbb{E}\left[e^{\int_{0}^{\tau_{n}} c\left(\xi_{n}(r), X_{n}(r)\right) d r}\left|G\left(t_{n}-\tau_{n}, X_{n}\left(\tau_{n}\right)\right)-G(t, x)\right|\right] \\
& +\mathbb{E}\left[|G(t, x)|\left|e^{\int_{0}^{\tau_{n}} c\left(\xi_{n}(r), X_{n}(r)\right) d r}-1\right|\right] .
\end{aligned}
$$

Because $\left|e^{\int_{0}^{\tau_{n}} c\left(\xi_{n}, X_{n}\right) d r}-1\right| \leq e^{c_{0}(t+\alpha)}+1$, we have that (4.71) is uniformly integrable and repeating the same argument made with (4.48), we can prove that

$$
\mathbb{E}\left[|G(t, x)|\left|e^{\int_{0}^{\tau_{n}} c\left(\xi_{n}(r), X_{n}(r)\right) d r}-1\right|\right] \underset{n \rightarrow \infty}{\longrightarrow} 0
$$

Next, we work with (4.70). As in estimate (4.42), we can prove that the sequence

$$
\left\{e^{\tau_{0}^{\tau_{n}} c\left(\xi_{n}(r), X_{n}(r)\right) d r}\left|G\left(t_{n}-\tau_{n}, X_{n}\left(\tau_{n}\right)\right)-G(t, x)\right|\right\}_{n \geq N_{1}}
$$

is uniformly integrable. We have that

$$
\begin{aligned}
(4.70) \leq & e^{c_{0}(t+\alpha)} \mathbb{E}\left[\left|G\left(t_{n}-\tau_{n}, X_{n}\left(\tau_{n}\right)\right)-G\left(t_{n}-\tau_{n}, X\left(\tau_{n}\right)\right)\right|\right] \\
& \left.+e^{c_{0}(t+\alpha)} \mathbb{E}\left[\mid G\left(t_{n}-\tau_{n}, X\left(\tau_{n}\right)\right)-G(t, x)\right) \mid\right] .
\end{aligned}
$$

We repeat the same arguments made for the estimates to (4.49) and (4.50) with (4.74) and (4.75), respectively. Then, we can prove that

$$
\begin{gathered}
\mathbb{E}\left[\left|G\left(t_{n}-\tau_{n}, X_{n}\left(\tau_{n}\right)\right)-G\left(t_{n}-\tau_{n}, X\left(\tau_{n}\right)\right)\right|\right] \underset{n \rightarrow \infty}{\longrightarrow} 0, \\
\left.\mathbb{E}\left[\mid G\left(t_{n}-\tau_{n}, X\left(\tau_{n}\right)\right)-G(t, x)\right) \mid\right] \underset{n \rightarrow \infty}{\longrightarrow} 0 .
\end{gathered}
$$

So, $v_{2} \in C([0, \infty) \times \bar{D})$ and the proof is complete. 


\subsection{Differentiability of $v$}

Let $0 \leq T_{0}<T_{1}$ and $A \subset D$ be a bounded, open, connected set with $C^{2}$ boundary. Consider the following Cauchy-Dirichlet problem:

$$
\begin{gathered}
-u_{t}(t, x)+\mathcal{L}[u](t, x)+c(t, x) u(t, x)=-f(t, x) \quad(t, x) \in\left(T_{0}, T_{1}\right] \times A, \\
u\left(T_{0}, x\right)=v\left(T_{0}, x\right) \quad \text { for } x \in A, \\
u(t, x)=v(t, x) \quad \text { for }(t, x) \in\left(T_{0}, T_{1}\right] \times \partial A,
\end{gathered}
$$

where the boundary data is $v$. If we assume $\mathbf{H} \mathbf{0}, \mathbf{H 1}$, and $\mathbf{H} \mathbf{2}$, then by the continuity of $v$ (Theorem 4.1) and Theorem 5.5, we can guarantee the existence of a unique classical solution to problem (4.77). To prove the regularity of $v$, we show that it coincides with the solution to (4.77) in the set $\left(T_{0}, T_{1}\right) \times A$ and so $v \in C^{1,2}\left(\left(T_{0}, T_{1}\right) \times A\right)$. Since $T_{0}, T_{1}$, and $A$ are arbitrary, we get the desired regularity. We are ready to prove the next theorem.

Theorem 4.4. Assume H0, H1, and H2. Let $v$ be defined as in (4.1). Then $v \in C_{\text {loc }}^{1,2, \lambda}\left((0, \infty) \times \mathbb{R}^{d}\right)$.

Proof. Let $w$ be the solution to (4.77). Define the following stopping times

$$
\begin{gathered}
\theta_{T}:=\inf \left\{s>0 \mid \xi(s)<T_{0}\right\} \\
\theta_{A}:=\inf \{s>0 \mid X(s) \notin \bar{A}\}, \\
\theta:=\theta_{T} \wedge \theta_{A} .
\end{gathered}
$$

Following the same arguments of Section 5 in Chapter 6 of [31], we can prove that $w$ has the following representation:

$$
w(t, x)=\mathbb{E}_{x}\left[\int_{0}^{\theta} e^{\int_{0}^{s} c(t-r, X(r)) d r} f(t-s, X(s)) d s\right]+\mathbb{E}_{x}\left[e^{\int_{0}^{\theta} c(t-r, X(r)) d s} v(t-\theta, X(\theta))\right] .
$$

Next, we prove that $v$ satisfies the following equality:

$$
v(t, x)=\mathbb{E}_{t, x}\left[\int_{0}^{\theta} e^{\int_{0}^{s} c(\xi(r), X(r)) d r} f(\xi(s), X(s)) d s\right]+\mathbb{E}_{t, x}\left[e^{\int_{0}^{\theta} c(\xi(r), X(r)) d s} v(\xi(\theta), X(\theta))\right] .
$$

Let $v_{1}$ and $v_{2}$ be defined as in (4.2) and (4.3), where $\tau:=t \wedge \tau_{D}$ is introduced to simplify the notation. We will use the following representation of $v_{1}$ and $v_{2}$ :

$$
\begin{gathered}
v_{1}(t, x)=\mathbb{E}_{t, x}\left[\int_{0}^{\tau} e^{\int_{0}^{s} c(\xi(r), X(r)) d r} f(\xi(s), X(s)) d s\right], \\
v_{2}(t, x)=\mathbb{E}_{t, x}\left[e^{\int_{0}^{\tau} c(\xi(r), X(r)) d r} G(\xi(\tau), X(\tau))\right] .
\end{gathered}
$$


First, we work with $v_{1}$

$$
\begin{aligned}
v_{1}(t, x)= & \mathbb{E}_{t, x}\left[\mathbb{E}\left[\int_{0}^{\tau} e^{\int_{0}^{s} c(\xi(r), X(r)) d r} f(\xi(s), X(s)) d s \mid \mathcal{F}_{\theta}\right]\right] \\
= & \mathbb{E}_{t, x}\left[\mathbb{E}\left[\int_{0}^{\theta} e^{\int_{0}^{S} c(\xi(r), X(r)) d r} f(\xi(s), X(s)) d s \mid \boldsymbol{F}_{\theta}\right]\right] \\
& +\mathbb{E}_{t, x}\left[\mathbb{E}\left[\int_{\theta}^{\tau} e^{\int_{0}^{s} c(\xi(r), X(r)) d r} f(\xi(s), X(s)) d s \mid \boldsymbol{F}_{\theta}\right]\right] .
\end{aligned}
$$

We study the addends of the right-hand side separately

$$
(4.82)=\mathbb{E}_{t, x}\left[\int_{0}^{\theta} e^{\int_{0}^{s} c(\xi(r), X(r)) d r} f(\xi(s), X(s)) d s\right] .
$$

For (4.83), we make a couple of changes of variables to get

$$
\begin{aligned}
(4.83) & =\mathbb{E}_{t, x}\left[\mathbb{E}\left[\int_{0}^{\tau-\theta} e^{\int_{0}^{s+\theta} c(\xi(r), X(r)) d r} f(\xi(s+\theta), X(s+\theta)) d s \mid \mathcal{F}_{\theta}\right]\right] \\
& =\mathbb{E}_{t, x}\left[e^{\int_{0}^{\theta} c(\xi(r), X(r)) d r} \mathbb{E}\left[\int_{0}^{\tau-\theta} e^{\left.\int_{0}^{s} c(\xi(r+\theta)), X(r+\theta)\right) d r} f(\xi(s+\theta), X(s+\theta)) d s \mid \mathcal{F}_{\theta}\right]\right] .
\end{aligned}
$$

Since $\theta<\tau_{D}$ and $\theta$ is bounded, we get that (see Remark 2.1)

$$
\begin{aligned}
\tau & =\inf \{s>0 \mid(\xi(s), X(s)) \notin[0, \infty) \times \bar{D}\} \\
& =\theta+\inf \{s>0 \mid(\xi(s+\theta), X(s+\theta)) \notin[0, \infty) \times \bar{D}\},
\end{aligned}
$$

so

$$
\tau-\theta=\Theta_{\theta} \circ \tau
$$

where $\Theta$. denotes the shift operator. Since the process $(\xi, X)$ is a homogeneous strong Markov process, we get that

$$
\begin{aligned}
\mathbb{E}\left[\int_{0}^{\Theta_{\theta} \circ \tau} e^{\int_{0}^{s} c\left(\Theta_{\theta} \circ(\xi, X)(r)\right) d r} f\left(\Theta_{\theta} \circ(\xi, X)(s)\right) d s \mid \mathcal{F}_{\theta}\right] \\
\quad=\mathbb{E}_{\xi(\theta), X(\theta)}\left[\int_{0}^{\tau} e^{\int_{0}^{s} c(\xi(r), X(r)) d r} f(\xi(s), X(s)) d s\right] \\
=v_{1}(\xi(\theta), X(\theta)) .
\end{aligned}
$$


The last equality follows from a general form of the strong Markov property (see Theorem 4.18 in Chapter 5 of [29] Theorem 4.6 in Chapter 5 of [27], or Theorem 5.1 in Chapter V of [26]) that states that if $F: \Omega \rightarrow \mathbb{R}$ is a bounded measurable functional, then

$$
\mathbb{E}\left[\left(F(\xi(\cdot), X(\cdot)) \circ \Theta_{\theta}\right) \mathbb{1}_{\{\theta<\infty\}} \mid \mathscr{F}_{\theta}\right]=\mathbb{E}_{\xi(\theta), X(\theta)}[F(\xi(\cdot), X(\cdot))]
$$

In both sides of (4.88), we consider the sequence $(F \vee-n) \wedge n$ and apply the conditional dominated convergence Theorem.

So,

$$
\begin{aligned}
v_{1}(t, x)= & \mathbb{E}_{t, x}\left[\int_{0}^{\theta} e^{\int_{0}^{s} c(\xi(r), X(r)) d r} f(\xi(s), X(s)) d s\right] \\
& +\mathbb{E}_{t, x}\left[e^{\int_{0}^{\theta} c(\xi(r), X(r)) d r} v_{1}(\xi(\theta), X(\theta))\right] .
\end{aligned}
$$

Next, we study $v_{2}$. Again, for the integral, we use a couple of changes of variables to get

$$
\begin{aligned}
v_{2}(t, x) & =\mathbb{E}_{t, x}\left[\mathbb{E}\left[e^{\int_{0}^{\tau} c(\xi(r), X(r)) d r} G(\xi(\tau), X(\tau)) \mid \mathcal{F}_{\theta}\right]\right] \\
& =\mathbb{E}_{t, x}\left[e^{\int_{0}^{\theta} c(\xi(r), X(r)) d r} \mathbb{E}\left[e^{\int_{0}^{\tau-\theta} c(\xi(r+\theta), X(r+\theta)) d r} G(\xi(\tau), X(\tau)) \mid \mathcal{F}_{\theta}\right]\right] .
\end{aligned}
$$

Using (4.87), we write

$$
\begin{aligned}
G(\xi(\tau), X(\tau)) & =G(\xi(\tau-\theta+\theta), X(\tau-\theta+\theta)) \\
& =G\left(\xi\left(\Theta_{\theta} \circ \tau+\theta\right), X\left(\Theta_{\theta} \circ \tau+\theta\right)\right) .
\end{aligned}
$$

Then, the expression inside the conditional expectation can be written as

$$
e^{\int_{0}^{\Theta_{\theta} \circ \tau} c\left(\Theta_{\theta} \circ(\xi, X)(r)\right) d r} G\left(\Theta_{\theta} \circ(\xi, X)\left(\Theta_{\theta} \circ \tau\right)\right)
$$

Repeating the same argument used for (4.88), we have that the conditional expectation is

$$
\begin{aligned}
\mathbb{E}\left[e^{\int_{0}^{\tau} c(\xi(r), X(r)) d r} G(\xi(\tau), X(\tau)) \mid \mathcal{F}_{\theta}\right] \\
\quad=\mathbb{E}\left[e^{\int_{\Theta_{\theta} \circ \tau} c\left(\Theta_{\theta} \circ(\xi, X)(r)\right) d r} G\left(\Theta_{\theta} \circ(\xi, X)\left(\Theta_{\theta} \circ \tau\right)\right) \mid \mathcal{F}_{\theta}\right] \\
=\mathbb{E}_{\xi(\theta), X(\theta)}\left[e^{\int_{0}^{\tau} c(\xi(r), X(r)) d r} G(\xi(\tau), X(\tau))\right] .
\end{aligned}
$$


Finally we get

$$
\begin{aligned}
v_{2}(t, x) & =\mathbb{E}_{t, x}\left[e^{\int_{0}^{\theta} c(\xi(r), X(r)) d r} \mathbb{E}_{\xi(\theta), X(\theta)}\left[e^{\int_{0}^{\tau} c(\xi(r), X(r)) d r} G(\xi(\tau), X(\tau))\right]\right] \\
& =\mathbb{E}_{t, x}\left[e^{\int_{0}^{\theta} c(\xi(r), X(r)) d r} v_{2}(\xi(\theta), X(\theta))\right] .
\end{aligned}
$$

Combining equations (4.90) and (4.95) we prove that (4.80) holds.

So due to equations (4.79) and (4.80) we have that $v=w$. Since $w \in C^{1,2, \lambda}\left(\left(T_{0}, T_{1}\right) \times A\right)$ (see Theorem 5.5 below) and $T_{0} T_{1}$ and $A$ are arbitrary we get that $v \in C^{1,2}\left((0, \infty) \times \mathbb{R}^{d}\right) \cap$ $C_{\text {loc }}^{1,2, \lambda}\left((0, \infty) \times \mathbb{R}^{d}\right)$ and the proof is complete.

We are ready to proof the Main Theorem

Proof of Theorem 3.1. The proof follows from Theorems 4.1 and 4.4 and Lemmas 3.3 and 3.4.

\section{Auxiliary Results}

\subsection{Continuity of the Stopping Times}

Theorem 5.1. Let $\{Z(t)\}_{t \geq 0}$ be a stochastic process with continuous paths a.s. and $A \subset \mathbb{R}^{d}$ an open, connected set with regular boundary. Let

$$
\tau:=\inf \{t>0 \mid Z(t) \notin \bar{A}\}
$$

Assume that $\mathbb{P}[\tau<\infty \mid Z(0)=z]=1$ and $\mathbb{P}\left[\tau=\tau^{\prime} \mid Z(0)=z\right]=1$ for all $z \in A$, where

$$
\tau^{\prime}:=\inf \{t>0 \mid Z(t) \notin A\} .
$$

For $a>0$, define

$$
\begin{gathered}
A_{a}:=\left\{x \in \mathbb{R}^{d} \mid d(x, \partial A)<a\right\}, \\
A_{a_{+}}:=A \cup A_{a}, \\
A_{a_{-}}:=A \backslash A_{a}
\end{gathered}
$$

and the corresponding exit times

$$
\begin{gathered}
\tau_{a+}:=\inf \left\{t>0 \mid Z(t) \notin \overline{A_{a+}}\right\}, \\
\tau_{a_{-}}:=\inf \left\{t>0 \mid Z(t) \notin \overline{A_{a-}}\right\} .
\end{gathered}
$$


Then, if $Z(0)=z \in A$, then

$$
\begin{gathered}
\tau_{a+} \underset{a \downarrow 0}{\stackrel{a . s .}{.}} \tau, \\
\tau_{a-} \frac{a . s .}{a \downarrow 0} \tau .
\end{gathered}
$$

Proof. Let $Z(0)=z \in A$ and

$$
B_{z}:=\{Z(t) \text { is continuous }\} \cap\left\{\tau=\tau^{\prime}\right\} \cap\{\tau<\infty\} .
$$

By the hypotheses, we have that $\mathbb{P}\left[B_{z}\right]=1$. Observe that $\tau_{a_{-}} \leq \tau \leq \tau_{a_{+}}$for all $a>0$, then we need to prove that, for all $\omega \in B_{z}$ and $\alpha>0$, there exists $\gamma(\omega, \alpha)>0$ such that, for all $0<a<\gamma$,

$$
\begin{aligned}
& 0 \leq \tau_{a+}(\omega)-\tau(\omega)<\alpha \\
& 0 \leq \tau(\omega)-\tau_{a-}(\omega)<\alpha
\end{aligned}
$$

Let $\omega \in B_{z}$ and $\alpha>0$, then $Z(t, \omega)$ is continuous and $\tau(\omega)<\infty$. We first prove the continuity for $\tau_{a+}$. Define

$$
\gamma_{+}(\alpha, \omega):=\sup _{\{t \in[\tau(\omega), \tau(\omega)+\alpha), Z(t, \omega) \notin \bar{A}\}}\{d(Z(t, \omega), \partial A)\}
$$

Since $Z(t, \omega)$ is continuous, then $\gamma_{+}(\alpha, \omega)>0$. So, there exists $t_{+} \in[\tau(\omega), \tau(\omega)+\alpha)$ such that $Z\left(t_{+}, \omega\right) \notin A_{a+}$ for all $0<a \leq\left(\gamma_{+} / 2\right)$. Let $\gamma:=\gamma_{+} / 2$, then, for all $0<a \leq \gamma$, we get that $\tau_{a+}(\omega) \in[\tau(\omega), \tau(\omega)+\alpha)$ and so

$$
0 \leq \tau_{a+}(\omega)-\tau(\omega)<\alpha
$$

For $\tau_{a^{-}}$, we proceed in a similar way. Let

$$
\beta(\omega):=\inf _{t \leq \tau(\omega)-\alpha}\{d(Z(t, \omega), \partial A)\}
$$

Since $\tau(\omega)=\tau^{\prime}(\omega)$ and $Z(t, \omega)$ is continuous, we get that $\beta(\omega)>0$. Define

$$
\gamma_{-}(\alpha, \omega):=\sup _{t \in(\tau(\omega)-\alpha, \tau(\omega)]}\{d(Z(t, \omega), \partial A)\}
$$

Again, it follows from the continuity of $Z(t, \omega)$ that $\gamma_{-}(\alpha, \omega)>0$. So, there exists $t_{-} \in(\tau(\omega)-$ $\alpha, \tau(\omega)]$ such that $Z\left(t_{-}, \omega\right) \notin A_{a-}$ for all $0<a \leq\left(\beta \wedge \gamma_{-}\right) / 2$. Let $\gamma:=\left(\beta \wedge \gamma_{-}\right) / 2$, then, for all $0<a \leq \gamma$, we get that $\tau_{a-}(\omega) \in(\tau(\omega)-\alpha, \tau(\omega)]$ and so

$$
0 \leq \tau(\omega)-\tau_{a-}(\omega)<\alpha
$$

and the proof is complete. 
Remark 5.2. For the continuity of $\tau_{a_{-}}$, we need the extra constant $\beta$. If we only consider the constant $\gamma_{-}$, then we can guarantee that the process $Z$ is "close" to $\partial A$ in the interval $(\tau-\alpha, \tau]$ but we cannot guarantee that it is the first time that it does it and so we do not necessarily have that $\tau_{a-}$ and $\tau$ are "close enough."

Theorem 5.3. Let $\{Z(t)\}_{t \geq 0}$ be a stochastic process with $Z(0)=z \in \mathbb{R}^{d}$ and $A \subset \mathbb{R}^{d}$ an open, connected set with regular boundary. Let $\left\{z_{n}\right\}$ be a sequence such that

$$
z_{n} \underset{n \rightarrow \infty}{\longrightarrow} z_{0}
$$

with $z_{n}, z_{0} \in A$. Denote by $Z_{0}$ and $Z_{n}$ the stochastic processes with initial conditions $z_{0}$ and $z_{n}$, respectively. Define

$$
\begin{gathered}
\tau:=\inf \left\{t>0 \mid Z_{0}(t) \notin \bar{A}\right\}, \\
\tau_{n}:=\inf \left\{t>0 \mid Z_{n}(t) \notin \bar{A}\right\} .
\end{gathered}
$$

Assume that $\tau<\infty$ and $\tau=\tau^{\prime}$ a.s., where

$$
\tau^{\prime}:=\inf \left\{t>0 \mid Z_{0}(t) \notin A\right\} .
$$

Assume also that, for all $T>0$,

$$
\left\|Z_{n}-Z\right\|_{T} \underset{n \rightarrow \infty}{\stackrel{\mathbb{P}}{\longrightarrow}} 0
$$

Then,

$$
\tau_{n} \underset{n \rightarrow \infty}{\stackrel{\mathbb{P}}{\longrightarrow}} \tau
$$

Proof. Let $\epsilon>0$ and $\eta>0$. We need to prove that there exists $N(\epsilon, \eta) \in \mathbb{N}$ such that, for all $n \geq N(\epsilon, \eta)$, we have

$$
\mathbb{P}\left[\left|\tau_{n}-\tau\right| \leq \epsilon\right] \geq 1-\eta
$$

Since $\tau<\infty$ a.s., then there exists $M=M(\eta)$ such that

$$
\mathbb{P}[\tau \leq M] \geq 1-\frac{\eta}{3}
$$

Thanks to Theorem 5.1, we get the existence of $a_{0}=a_{0}(\epsilon, \eta)$ such that, for all $a \leq a_{0}(\epsilon, \eta)$, it is satisfied that

$$
\mathbb{P}\left[\tau_{a+} \leq \tau+\epsilon, \tau_{a-} \geq \tau-\epsilon\right] \geq 1-\frac{\eta}{3} .
$$


Let $a_{1}:=a_{0} / 2$. By hypothesis, we have the existence of $N=N\left(M, a_{1}, \epsilon, \eta\right)=N(\epsilon, \eta)$ such that for all $n \geq N(\epsilon, \eta)$

$$
\mathbb{P}\left[\left\|Z_{n}-Z\right\|_{M+\epsilon} \leq a_{1}\right] \geq 1-\frac{\eta}{3} .
$$

So, over the intersection of the three sets, we get

$$
\begin{aligned}
\{\tau \leq M\} & \cap\left\{\tau_{a_{1}+} \leq \tau+\epsilon, \tau_{a_{1}-} \geq \tau-\epsilon\right\} \cap\left\{\left\|Z_{n}-Z\right\|_{M+\epsilon} \leq a_{1}\right\} \\
& \subset\left\{\tau_{a_{1^{-}}} \leq \tau_{n} \leq \tau_{a_{1^{+}}}\right\} \cap\left\{\tau_{a_{1}+} \leq \tau+\epsilon, \tau_{a_{1^{-}}} \geq \tau-\epsilon\right\} \\
& \subset\left\{\tau-\epsilon \leq \tau_{n} \leq \tau+\epsilon\right\}
\end{aligned}
$$

The first inclusion follows from the fact that, over the set $\{\tau \leq M\} \cap\left\{\left\|Z_{n}-Z\right\|_{M+\varepsilon} \leq a_{1}\right\}$, we have that the process $Z_{n}$ cannot leave the set $A$ before the process $Z$ leaves the set $A_{a_{1}-}$ and so we get the $\tau_{a_{1}-} \leq \tau_{n}$. On the intersection of the three sets, we have that $\tau_{a_{1}+} \leq M+\epsilon$. Considering a similar argument, we obtain the remaining part, that is, $\tau_{n} \leq \tau_{a_{1}+}$.

Finally, we get from inequalities (5.19), (5.20), (5.21) and Bonferroni's inequality that for all $n \geq N(\epsilon, \eta)$,

$$
\mathbb{P}\left[\tau-\epsilon \leq \tau_{n} \leq \tau+\epsilon\right] \geq 1-\eta
$$

and the proof is complete.

\subsection{Additional Results}

Theorem 5.4. Let $\left\{\left(t_{n}, x_{n}\right)\right\}_{n \in \mathbb{N}} \subset[0, \infty) \times \mathbb{R}^{d}$ be a sequence such that $\left(t_{n}, x_{n}\right) \underset{n \rightarrow \infty}{\rightarrow}(t, x)$. Denote by $X_{n}$ and $X$ the solutions of the following equations:

$$
\begin{gathered}
d X_{n}(s)=b\left(t_{n}-s, X_{n}(s)\right) d s+\sigma\left(t_{n}-s, X_{n}(s)\right) d W(s), \quad X_{n}(0)=x_{n}, \\
d X(s)=b(t-s, X(s)) d s+\sigma(t-s, X(s)) d W(s), \quad X(0)=x .
\end{gathered}
$$

Then, for all $T>0$,

$$
\left\|X_{n}-X\right\|_{T} \underset{n \rightarrow \infty}{\stackrel{\mathbb{P}}{\longrightarrow}} 0
$$

Proof. This theorem is consequence of Theorem 1.5 in Chapter V of [26].

The following theorem is Theorem 9 of Chapter 3 in [3]. Let $0 \leq T_{0}<T_{1}$ and let $A \subset \mathbb{R}^{d}$ be a bounded open set with $C^{2}$ boundary. Since $\sigma, b, c$, and $f$ are locally Lipschitz, then they are locally Hölder of any order $\beta \in(0,1)$ 
Theorem 5.5. Assume $\mathbf{H 1}$ and $\mathbf{H 2}$. Consider the following differential equation:

$$
\begin{gathered}
-u_{t}(t, x)+\mathcal{L}[u](t, x)+c(t, x) u(t, x)=-f(t, x) \quad(t, x) \in\left[T_{0}, T_{1}\right] \times A, \\
u\left(T_{0}, x\right)=g\left(T_{0}, x\right) \text { for } x \in A, \\
u(t, x)=g(t, x) \quad \text { for }(t, x) \in\left(T_{0}, T_{1}\right] \times \partial A .
\end{gathered}
$$

If $g$ is continuous, then there exists a classical solution $w \in C\left(\left[T_{0}, T_{1}\right) \times \bar{A}\right) \cap C^{1,2, \beta}\left(\left(T_{0}, T_{1}\right) \times A\right)$ of (5.26).

Remark 5.6. Let $w$ be the solution of (5.26) and define $z$ as $w(t, x)=e^{c_{0} t} z(t, x)$ in $\left[T_{0}, T_{1}\right] \times A$. Then, $z$ fulfils (5.26) with $c^{\prime}=c-c_{0}$ and $f^{\prime}(t, x)=e^{-c_{0} t} f(t, x)$. And so the hypotheses of Theorem 9 of Chapter 3 in [3] are satisfied.

\section{Acknowledgments}

The author would like to thank specially Begoña Fernández for her valuable comments which helped in developing the results and improving the presentation of this paper. The author's thanks also go to Daniel Hernández, Ana Meda, Luz de Teresa, and Ekaterina Todorova for all the discussions on the subject which have been very fruitful and stimulating. The assistance by Nelson Muriel in the presentation of the paper is gratefully acknowledged. Finally, The author most sincere gratitude to the reviewers for their very appropriate and precise remarks. The suggested improvements in precision and corrections substantially improved the quality of the present paper. This work was partially supported by Grant nos. PAPIIT-DGAPAUNAM IN103660, IN117109, and CONACYT 180312, México.

\section{References}

[1] G. M. Lieberman, Second Order Parabolic Differential Equations, World Scientific, River Edge, NJ, USA, 1996.

[2] O. A. Ladyženskaja, V. A. Solonnikov, and N. N. Uralceva, Linear and Quasilinear Equations of Parabolic Type, Translations of Mathematical Monographs, Vol. 23, American Mathematical Society, Providence, RI, USA, 1967, Translated from the Russian by S. Smith.

[3] A. Friedman, Partial Differential Equations of Parabolic Type, Prentice-Hall, Englewood Cliffs, NJ, USA, 1964.

[4] A. Lunardi, Analytic Semigroups and Optimal Regularity in Parabolic Problems, Progress in Nonlinear Differential Equations and Their Applications 16, Birkhäuser, Basel, Switzerland, 1995.

[5] M. Freidlin, Functional Integration and Partial Differential Equations, vol. 109 of Annals of Mathematics Studies, Princeton University Press, Princeton, NJ, USA, 1985.

[6] D. G. Aronson and P. Besala, "Parabolic equations with unbounded coefficients," Journal of Differential Equations, vol. 3, pp. 1-14, 1967.

[7] P. Besala, "On the existence of a fundamental solution for a parabolic differential equation with unbounded coefficients," Annales Polonici Mathematici, vol. 29, no. 4, pp. 403-409, 1975.

[8] G. Da Prato and A. Lunardi, "On the Ornstein-Uhlenbeck operator in spaces of continuous functions," Journal of Functional Analysis, vol. 131, no. 1, pp. 94-114, 1995. 
[9] S. Cerrai, "Elliptic and parabolic equations in $\mathrm{R}^{n}$ with coefficients having polynomial growth," Communications in Partial Differential Equations, vol. 21, no. 1-2, pp. 281-317, 1996.

[10] A. Lunardi and V. Vespri, "Optimal $L^{\infty}$ and Schauder estimates for elliptic and parabolic operators with unbounded coefficients," in Reaction Diffusion Systems, vol. 194 of Lecture Notes in Pure and Applied Mathematics, pp. 217-239, Dekker, New York, NY, USA, 1998.

[11] S. Cerrai, "Some results for second order elliptic operators having unbounded coefficients," Differential and Integral Equations, vol. 11, no. 4, pp. 561-588, 1998.

[12] A. Lunardi, "Schauder theorems for linear elliptic and parabolic problems with unbounded coefficients in $\mathrm{R}^{n}$," Studia Mathematica, vol. 128, no. 2, pp. 171-198, 1998.

[13] G. Da Prato and B. Goldys, "Elliptic operators on $\mathbb{R}^{d}$ with unbounded coefficients," Journal of Differential Equations, vol. 172, no. 2, pp. 333-358, 2001.

[14] E. Priola, "The Cauchy problem for a class of Markov-type semigroups," Communications in Applied Analysis, vol. 5, no. 1, pp. 49-75, 2001.

[15] T. Deck and S. Kruse, "Parabolic differential equations with unbounded coefficients—a generalization of the parametrix method," Acta Applicandae Mathematicae, vol. 74, no. 1, pp. 71-91, 2002.

[16] G. Metafune, D. Pallara, and M. Wacker, "Feller semigroups on $\mathrm{R}^{N}$," Semigroup Forum, vol. 65, no. 2, pp. 159-205, 2002.

[17] M. Bertoldi and L. Lorenzi, "Estimates of the derivatives for parabolic operators with unbounded coefficients," Transactions of the American Mathematical Society, vol. 357, no. 7, pp. 2627-2664, 2005.

[18] S. Fornaro, G. Metafune, and E. Priola, "Gradient estimates for Dirichlet parabolic problems in unbounded domains," Journal of Differential Equations, vol. 205, no. 2, pp. 329-353, 2004.

[19] M. Bertoldi and S. Fornaro, "Gradient estimates in parabolic problems with unbounded coefficients," Studia Mathematica, vol. 165, no. 3, pp. 221-254, 2004.

[20] M. Bertoldi, S. Fornaro, and L. Lorenzi, "Gradient estimates for parabolic problems with unbounded coefficients in non convex unbounded domains," Forum Mathematicum, vol. 19, no. 4, pp. 603-632, 2007.

[21] L. Lorenzi and M. Bertoldi, Analytical Methods for Markov Semigroups, vol. 283 of Pure and Applied Mathematics, Chapman \& Hall/CRC, Boca Raton, Fla, USA, 2007.

[22] G. Da Prato and A. Lunardi, “On a class of elliptic operators with unbounded coefficients in convex domains," Atti della Accademia Nazionale dei Lincei. Classe di Scienze Fisiche, Matematiche e Naturali. Rendiconti Lincei. Serie IX. Matematica e Applicazioni, vol. 15, no. 3-4, pp. 315-326, 2004.

[23] G. Da Prato and A. Lunardi, "On a class of elliptic and parabolic equations in convex domains without boundary conditions," Discrete and Continuous Dynamical Systems. Series A, vol. 22, no. 4, pp. 933-953, 2008.

[24] M. Geissert, H. Heck, M. Hieber, and I. Wood, "The Ornstein-Uhlenbeck semigroup in exterior domains," Archiv der Mathematik, vol. 85, no. 6, pp. 554-562, 2005.

[25] M. Hieber, L. Lorenzi, and A. Rhandi, "Second-order parabolic equations with unbounded coefficients in exterior domains," Differential and Integral Equations, vol. 20, no. 11, pp. 1253-1284, 2007.

[26] N. V. Krylov, Introduction to the Theory of Diffusion Processes, vol. 142 of Translations of Mathematical Monographs, American Mathematical Society, Providence, RI, USA, 1995, Translated from the Russian manuscript by Valim Khidekel and Gennady Pasechnik.

[27] R. Durrett, Stochastic Calculus, Probability and Stochastics Series, CRC Press, Boca Raton, Fla, USA, 1996.

[28] E. B. Dynkin, Diffusions, Superdiffusions and Partial Differential Equations, vol. 50 of American Mathematical Society Colloquium Publications, American Mathematical Society, Providence, RI, USA, 2002.

[29] C. Tudor, Proceso Estocástico, vol. 2 of Textos Aportaciones Matemáticas: Textos, Sociedad Matemática Mexicana, Guanojuato, México, 2002.

[30] D. W. Stroock and S. R. S. Varadhan, Multidimensional Diffusion Processes, Classics in Mathematics, Springer, Berlin, Germany, 2006.

[31] A. Friedman, Stochastic Differential Equations and Applications. Vol.1, Probability and Mathematical Statistics, Vol. 28, Harcourt Brace Jovanovich Publishers; Academic Press, New York, NY, USA, 1975. 
[32] R. M. Dudley, Real Analysis and Probability, vol. 74 of Cambridge Studies in Advanced Mathematics, Cambridge University Press, Cambridge, UK, 2002.

[33] A. Gut, Probability: A Graduate Course, Springer Texts in Statistics, Springer, New York, NY, USA, 2005. 


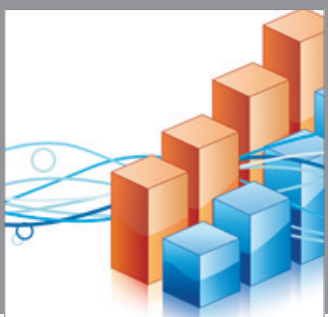

Advances in

Operations Research

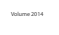

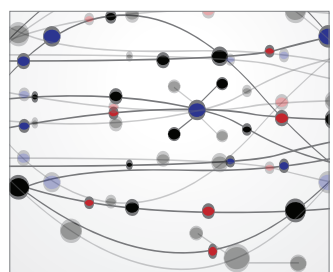

\section{The Scientific} World Journal
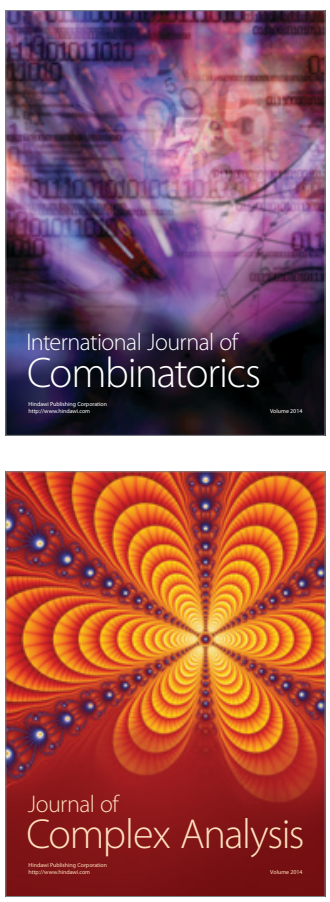

International Journal of

Mathematics and

Mathematical

Sciences
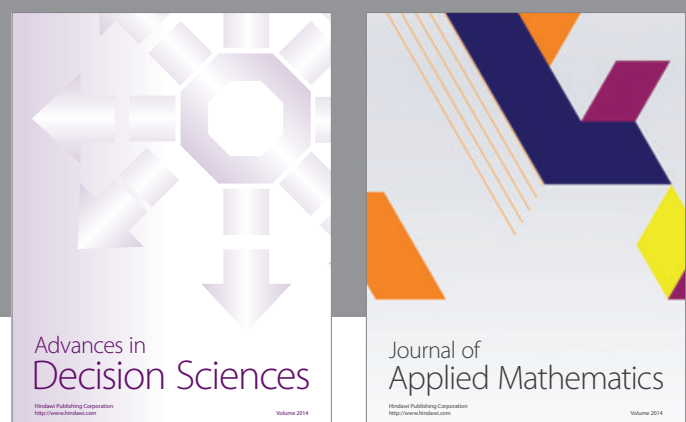

Journal of

Applied Mathematics
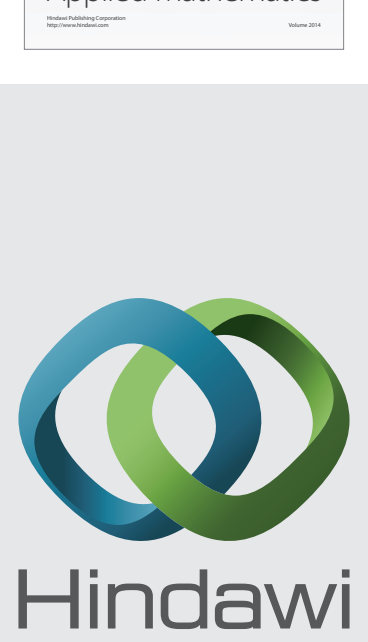

Submit your manuscripts at http://www.hindawi.com
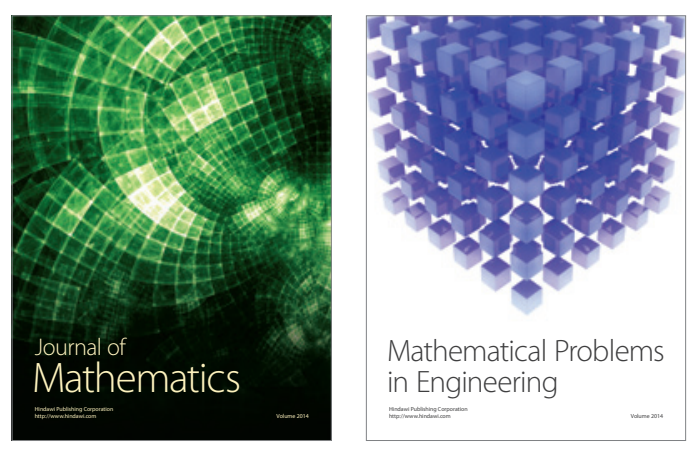

Mathematical Problems in Engineering
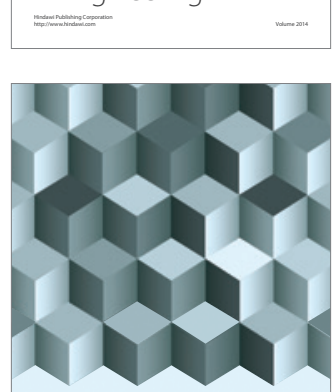

Journal of

Function Spaces
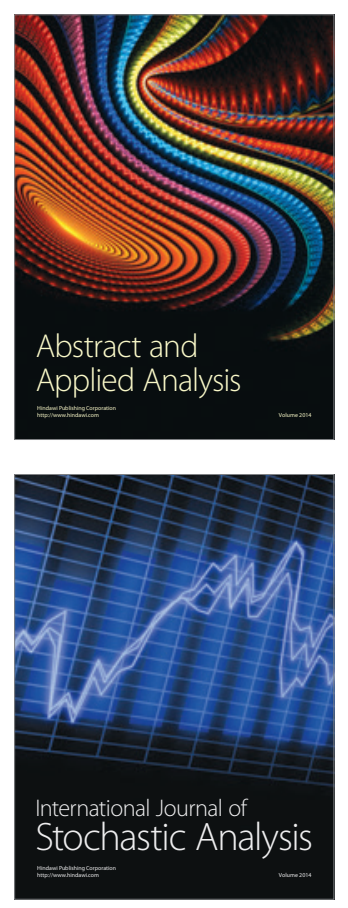

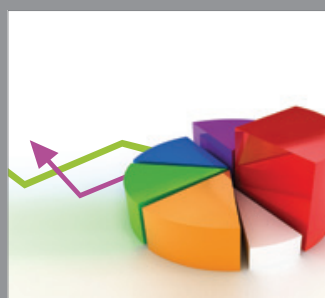

ournal of

Probability and Statistics

Promensencen
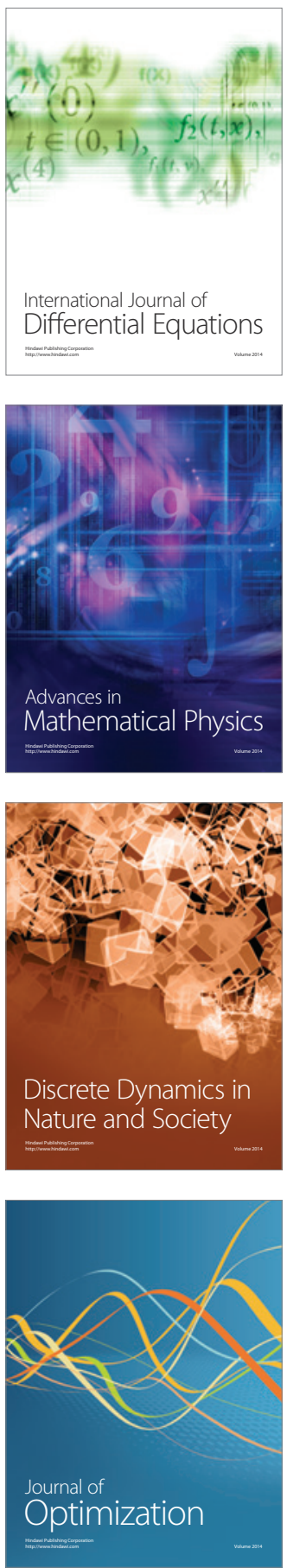